\title{
Inoculation with mycorrhizal fungi and irrigation management shaped the bacterial and fungal communities and networks in vineyard soils
}

Nazareth Torres

University of California Davis

Runze Yu

University of California Davis

Kurtural Kurtural ( $\nabla$ skkurtural@ucdavis.edu )

University of California Davis https://orcid.org/0000-0001-9578-831X

\section{Research}

Keywords: Arbuscular mycorrhizal fungi, co-occurrence networks, grapevine, soil health, water deficit

Posted Date: April 5th, 2021

DOI: https://doi.org/10.21203/rs.3.rs-322965/v1

License: (c) (i) This work is licensed under a Creative Commons Attribution 4.0 International License.

Read Full License 


\section{Abstract}

Background: Vineyard-living microbiota play a relevant role in supporting grapevine health and adaptation to changing environments and determining the biological quality of soils that strongly influence the wine quality and characteristics. However, management practices may shape the abundance and interactions of bacteria and fungi in vineyard soils. We conducted this experiment to assess whether the vineyard soil microbiome was altered by different management practices with the use of biostimulants (arbuscular mycorrhizal fungi (AMF) inoculation vs. non-inoculated) and/or irrigation management (full irrigated vs. half irrigated).

Results: Bacterial and fungal communities in vineyard soils were shaped by both time course and soil management (i.e. use of biostimulants or irrigation). Regarding alpha diversity, fungal communities were more responsive to treatments whereas changes on beta diversity were mainly recorded in the bacterial communities. Edaphic factors rarely influenced bacterial and fungal communities. Microbial network analyses suggested that the bacterial associations were weaker than the fungal ones under half irrigation and that the inoculation with AMF led to the increase of positive associations between vineyard soil-living microbes.

Conclusion: Our results indicated different sensibilities within microbial communities living in vineyards, as well as, how they changed during the season, and in response to management practices. Moreover, results highlighted the need of more studies leading with the effect of management practices, especially the addition of AMF, to cropping systems to fully understand the factors that drive their variability, strengthen beneficial microbial networks and achieved a better soil quality that improves crop performance.

\section{Background}

Plant microbiomes play an important role in supporting plant health and adaptation to changing environments [1]. The biological quality of soils may be defined as the capacity of a soil to host a large quantity and diversity of living organisms, which are involved in its functioning and in the provision of ecosystem services [2]. Within these services, the role of microorganisms on decomposition, mineralization of plant nutrients, atmospheric $\mathrm{N}$ fixation and $\mathrm{C}$ sequestration, is particularly relevant for cropping systems [3]. In vineyards, the composition of the soil microbiome has been recently highlighted because it seems to be the major driver in shaping the bacterial and fungal communities associated with grapevine tissues, including berries [4], defining the regional characteristics of the wine [5,6]. Thus, the traditional conception of 'terroir', defined as the interaction of the vine with its ecosystem [7], requires the addition of the 'microbial terroir' concept given that microbial vineyard inhabitants would determine regional grape characteristics and quality [8-11]. Indeed, it was recently highlighted the need to reconcile the 'terroir' and the soil health concepts in the context of wine production [3]. 
The effect of different viticulture management practices on grapevine physiology and berry composition is a recurrent topic in viticulture research. However, less is known about how the microbial communities' associations with plants and soil are affected. In this regard, previous studies reported that soil microbial diversity varied considerably between vineyards under conventional and ecological management, with bacterial communities strongly affected by tillage [12], whereas others demonstrated that land uses influenced the structure and composition of fungal communities and the geography affected bacterial communities [13].

Within management practices, irrigation is particularly relevant for grapevines due to many viticulture areas relies on irrigation for crop production [14]. Furthermore, the exacerbation of water deficit due to global warming is rapidly expanding irrigation in traditional rain fed viticulture regions to mitigate negative effects on grapevines [14]. However, irrigation practices are known to disturb the soil ecosystem, affecting soil characteristics and soil microbial functioning [15]. Furthermore, water availability in soils alters microbial communities with potentially long-term consequences, including ensuing plant community composition and the ability of aboveground and belowground communities to withstand future disturbances [16]. Despite these reports, the biological quality of winegrowing soils and the impact of viticultural practices are still poorly known and to the best of our knowledge, information is lacking about how water management may alter soil microbial communities.

Recent research suggested that plant symbionts strongly affect the plant-associated microbiome [17], however, little is known about how the "symbiosis cascade effects" proposed by these authors may shape soil microbial communities in crop production systems. Currently, over 400 microbial products are available in the markets as crop fertilizer or crop management tools [18]. The intensive practices of modern agriculture had detrimental environmental impact on soils such as, increased greenhouse gases emissions, nutrient leaching given the intensive fertilizer application, and a higher soil erosion and decreased biodiversity [2]. Therefore, searching environmental-friendly management practices is paramount to alleviate such deleterious effects. Previous studies suggested that biostimulants such as AMF inoculation might be used for enhancing plant resistance to abiotic environmental stresses by their effectiveness in improving crop productivity and quality under abiotic stresses [19]. However, few studies were conducted to assess their effect on plant-associated microbiota [20-22].

Therefore, understanding how management practices affect the soil microbiota is an important subject towards a more sustainable agriculture. We hypothesized that irrigation management may affect microbial diversity and soil chemical properties. Similarly, we aimed to study if AMF inoculation may promote the plant-growth promoting bacteria abundance while decreasing the abundance of pathogens in soils. Therefore, a randomized experiment was conducted to address the effect of irrigation management (half irrigation vs. full irrigation) and AMF inoculation (inoculated vs. non-inoculated) on bacterial and fungal communities living in the bulk soil of a newly established vineyard in its first productive year.

\section{Material And Methods}




\subsection{Site description and experimental design}

This study was conducted on a three-year old vineyard planted to Merlot (clone 181) grafted onto 3309C rootstock in 2020. Vines were planted at a density of $3 \mathrm{~m} \times 2 \mathrm{~m}$ (row $\times$ vine) and oriented East-West at the Oakville Experimental Research Station $\left(38.429^{\circ}-122.410^{\circ}\right)$ in California, USA. Natural vegetation was allowed to grow in alleys and mowed according to vineyard manager's discretion, but a no-till system was applied. In all sampling spots, grasses were mainly constituted from species of Poaceae family, Plantago $s p .$, Trifolium sp., Convulvulus sp. and Barbarea vulgaris. The experimental vineyard was drip-irrigated with emitters spaced every $2 \mathrm{~m}$ along the drip line.

Soil samples for microbial analysis were collected before treatment application (13 March) and after three months (16 June) in each treatment. The experiment was conducted in a split plot design with $2 \times 2$ factors (AMF inoculated or non-inoculated vines subjected to two irrigation amounts) in a randomized complete block (Additional file: Figure S1). Each experimental replicate consisted of 15 grapevines occupying $90 \mathrm{~m}^{2} /$ treatment-replicate.

AMF inoculated vines (inoculated, I) were drench in their trunk for 50 seconds with a diluted AMF inoculum at a $10 \mathrm{~g} / 1000$ plants rate following the manufacturer's recommendations by using a spot sprayer. The commercial Myco Apply Endo Maxx inoculum (Mycorrhizal Applications LLC, OR, USA) consisted of a suspendable powder containing living propagules of Rhizophagus intraradices (basionym Glomus intraradices), Funneliformis mosseae (basionym Glomus mosseae), Glomus aggregatum and Glomus etunicatum containing 5,625 propagules/g. In order to restore the rhizobacteria and other soil free-living microorganism accompanying AMF, non-inoculated vines (non-inoculated, $\mathrm{NI}$ ) received the same amount of a filtrate inoculum obtained by passing diluted mycorrhizal inoculum through a Whatman filter paper Grade 5 with particle retention of $2.5 \mu \mathrm{m}$ (Whatman 5; GE Healthcare, MA, USA). AMF inoculum and filtrate application occurred at the beginning of the growing season (March 20). Vineyard crop evapotranspiration ( $\mathrm{ET}_{\mathrm{c}}$ ) was calculated by multiplying the reference evapotranspiration ( $E T_{0}$, California Irrigation Management Information System, CIMIS \#77) and the crop coefficient $\left(K_{c}\right)$. Then, half of the I and NI plants were weekly irrigated (starting in 8 May), with the amount of water to keep the full of expansive growth (Full irrigated, $\mathrm{FI}$ ). Fl irrigation coincided with the amount of water needed to restore the $100 \%$ of the $\mathrm{ET}_{\mathrm{C}}$, while the other half of I and $\mathrm{NI}$ plants received half of the water amount of the $\mathrm{Fl}$ vines (half irrigated, $\mathrm{HI}$ ).

\subsection{Soil sampling, DNA extraction and sequencing}

Bulk soil samples for the microbiome analysis were collected at two time points (before and after treatment application) according to the established protocol [23]. Before treatment, soil samples (not treated, NT) were collected in the same plots in where treatments were then applied. Soil samples were collected at a depth of $15-20 \mathrm{~cm}$ and a distance of $30 \mathrm{~cm}$ away from the vine trunks, compiling four different cores within the plot. Each sample from a single plot and time point was made polling together with the top soil from four random spots in each plot and placed in sterile tubes and homogenized. 
Between each sampling point, digging tools were sterilized with $70 \%$ ethanol in order to avoid crosscontamination between plots.

\subsection{Edaphic factors of the plots subjected to different irrigation amounts and AMF inoculation.}

After treatment application (30 July), samples of soils were collected at a depth of $20 \mathrm{~cm}$. A cylindrical sample of $378.67 \mathrm{~cm}^{3}$ of bulk soil per sample was used to determine the soil bulk density and the soil water content (SWC). Soil samples were weighted and dried in an oven at $70^{\circ} \mathrm{C}$. Dried samples were used for determining the SWD and $\mathrm{BD}$ according to the following equations:

$\operatorname{SWC}(g / g)=\frac{\text { weight of moist soil - weight of oven dried soil }}{\text { weight of oven dried soil }}$

Bulk density $\left(g / \mathrm{cm}^{3}\right)=\frac{\text { weight of oven dried soil }}{\text { volume of soil }}$

20 grams of soil samples diluted in deionized water $(2: 5$, w:v) were used for determining the soil $\mathrm{pH}$ with an autotitrator (Omnis titrator, Methohm, Switzerland). Soil relative humidity $(\mathrm{RH})$, temperature and water evaporation from soils were measured in situ with a Soil Respiration Chamber (SRC-2) coupled to Ciras-3 system (PP Systems, Amesbury, MA, USA) at noon on June 9. The SRC-2 chamber was stabilized for one minute and then, parameters were recorded.

\subsection{Statistical analysis}

All the statistical analysis were carried out using the R-Studio version 3.6.1 (RStudio: Integrated Development for R., Boston, MA, USA). The R script and all necessary input files are provided as Additional file 2. Venn diagrams were generated using the package 'VennDiagram' for R [26]. Then, OTUs present in less than two thirds of the replicates were discarded to ensure reproducibility [27] before the statistical analyses. Alpha diversity indices (Observed richness, Shannon and Inverse Simpson) were calculated and fit in linear mixed-effect models (LMEM) by using the Imer function from '/me4' package [28] with not treated (NT), or different treatment, based on irrigation amounts and AMF inoculation (FINI, FII, HINI or HII), as fixed factors, and replicate as random factor [29]. The significance of the models was tested with the 'ImerTest' package [30]. Then, pairwise contrasts were conducted with function Ismeans from 'Ismeans'package [31] using the Kenward-Roger method and Tukey adjustment for p-values. OTUs table was considered to perform the Permutational Analysis Of Variance (PERMANOVA) and Permutational Analysis of Multivariate Homogeneity of groups dispersions (BETA-DISPER) using the functions Adonis and betadisper in the 'vegan' package [32] with 999 permutations. Similarities or dissimilarities of the studied communities, those which resulted significant from the PERMANOVA analysis, were plotted by Non-Metric Multidimensional Scaling Analysis (NMDS) and Principal Coordinates Analysis (PCoA). Differences in the relative abundance at the phylum and class levels between different treatments were assessed with LMEM as described above. Cladograms of the taxonomy were drawn using the R package 'Metacoder'[33]. The size of the nodes was proportional to 
the relative abundance of the taxa while color represents changes in the different plots. Soil chemical and physical parameters were analyzed by LMEM with AMF inoculation (M), irrigation treatment (I), and their combination $(\mathrm{M} \times \mathrm{I})$ as fixed factors, and replicate as random factor, then, the significance of the model and the contrasts between the treated plots were tested as described above. The influence of soil chemical and physical parameters on the microbial communities was explored by canonical correspondence analysis (CCA) using 'vegan' [32] and 'labdsv' [34] packages. Then, the significance of the CCA was evaluated with ANOVA. Finally, two networks were constructed based on time of sampling.

Thus, a co-ocurrence analysis of all treated plots/replicated was conducted before and after treatment (n $=16$ per each condition). Then, fungal and bacterial networks were separately constructed in each factor plot after treatment (FINI, FII, HINI or HII). In every network, all replicates were considered $(n=8)$ to obtain a more accurate correlation between different OTUs. Co-occurrence networks were analyzed by using the 'cooccur' package in $\mathrm{R}$ [35]. This method employs a probabilistic approach to determine which species pairs co-occur more (positive co-occurrence) or less often (negative co-occurrence) than expected. The analysis was conducted on the probabilistic model developed by Veech [36] based on pairwise comparisons of species presence/absence using primarily randomization (null model). Co-occurrence networks visualization was conducted by using the 'VisNetwork' package [37] with the same software.

\section{Results}

\subsection{Richness and diversity of bacterial and fungi communities in vineyard plots before and after AMF inoculation and irrigation systems application.}

Soil samples were collected from 16 plots in the Oakville vineyard before and after the treatment application (Additional file 1: Figure S1). Each treatment was characterized by the combination of mycorrhizal inoculation (I or $\mathrm{NI}$ ) and irrigation systems ( $\mathrm{Fl}$ or $\mathrm{HI}$ ) with four replicates (see "Material and Methods" section). Three kingdoms were identified in the samples (fungi, archaea and bacteria) (Additional file 3). Venn diagrams were generated before removing the less common species found in the trial (see "Material and Methods" section) to assess the distinct and common bacterial and fungal species among different treatments (Additional file 1: Figure S2). There were 97 bacterial and 273 fungal OTUs specific to the NT plots. On the other hand, there were 9 bacterial species were specific to FINI and HII whereas 19 bacterial OTUs were found in HINI as well as in Fll plots. Surprisingly, no specific fungal OTUs for plots after treatments were found. Thus, the number of bacterial species shared by all the plots were the $46.4 \%$ whereas for fungi accounted for the $21.4 \%$ of the total observations.

The analysis of a-diversity indices in different plots showed differences according to the LMEM (Figure 1). Regarding bacterial communities, species richness was decreased in the FINI plots comparing to NT ones (Figure 1A). However, no differences were found between the different plots regarding the Shannon or the inverse of Simpson indices (Figure $1 \mathrm{C}$ and $\mathrm{E}$ ). Fungal communities were significantly affected by the treatments. Thus, the number of fungal species identified was significantly lower after treatment application, especially in the Fll plots (Figure 1B). Shannon index was decreased after treatment in the NI 
plots (Figure 1D). On the other hand, inverse of Simpson index increased after treatment application in all the plots (Figure 1F).

In order to find possible effects of competition between bacteria and fungi, correlation between a-diversity indices was conducted. Pearson correlation across all samples was negligible for Shannon and inverse of Simpson indices $(R=0.16$, $p$ value $=0.39$ and $R=-0.095, p$ value $=0.6$, respectively) (Additional file 1 : Figure S3A and B). Similarly, relationships of the diversity indices within each treatment/ plots was insignificant suggesting no competition between fungal and bacterial communities (Additional file 4: Table S1).

Correlations between microbiome structure and composition and the applied treatments were studied by computing the between-sample diversity using Bray-Curtis distance (Figure 2). For bacteria, the Principal Coordinates Analysis (PCoA) showed dissimilarities between samples from different treatments that clustered separately in three groups, NT, the full irrigated plots (FINI and FII) and half irrigated (HINI and HII), respectively (Figure 2A). The first two components explained the $28.1 \%$ and the $18.8 \%$ of the variation. Additionally, all the applied treatments (AMF inoculation and irrigation amounts), time of sampling and their combination affected the bulk soil bacterial communities as showed Table 1. The ordination of the data in a non-metric multidimensional scaling (NMDS) indicated that all samples clustered closely suggesting similar bacterial communities (Figure 2B). The application of treatments significantly affected beta-diversity where PERMANOVA test showed a clear distinction ( $F=4.879$, $\mathrm{p}=0.001)$.

Table 1. $F$ and $p$ values of PERMANOVAs comparing different irrigation systems (I), AMF inoculation (M) and time course $(T)$ of Merlot vineyard microbial beta diversity. 


\begin{tabular}{|c|c|c|c|c|c|}
\hline \multirow[b]{3}{*}{ Treatment } & \multirow[b]{3}{*}{ Comparison } & \multicolumn{4}{|c|}{ Bray-Curtis } \\
\hline & & \multicolumn{2}{|c|}{ Bacteria } & \multicolumn{2}{|l|}{ Fungi } \\
\hline & & $\mathrm{F}$ & $\begin{array}{l}\mathrm{p} \\
\text { value }\end{array}$ & $\mathrm{F}$ & $\begin{array}{l}\mathrm{p} \\
\text { value }\end{array}$ \\
\hline Irrigation (I) & FI vs. HI & 4.727 & 0.001 & 6.480 & 0.001 \\
\hline $\begin{array}{l}\text { AMF } \\
\text { inoculation } \\
\text { (M) }\end{array}$ & NI vs. I & 3.528 & 0.002 & 5.446 & 0.005 \\
\hline $\begin{array}{l}\text { Time course } \\
(T)\end{array}$ & T0 vs. T1 & 9.688 & 0.001 & 26.762 & 0.001 \\
\hline$I \times M$ & FINI vs. Fll vs. HINI vs. HII & 3.424 & 0.001 & 4.598 & 0.001 \\
\hline $\mathrm{I} \times \mathrm{T}$ & FI_T0 vs. FI_T1 vs. HI_T0 vs. HI_T1 & 8.788 & 0.001 & 14.134 & 0.001 \\
\hline $\mathrm{M} \times \mathrm{T}$ & I_T0 vs. I_T1 vs. NI_T0 vs. NI_T1 & 5.312 & 0.001 & 14.092 & 0.001 \\
\hline $\mathrm{I} \times \mathrm{M} \times \mathrm{T}$ & $\begin{array}{l}\text { FINI_T0 vs. FINI_T1 vs. HINI_TO vs. HINI_T1 vs. } \\
\text { FII_T0 vs. FII_T1 vs. HII_TO vs. HII_T1 }\end{array}$ & 4.879 & 0.001 & 7.615 & 0.001 \\
\hline
\end{tabular}

On the other hand, the PCoA for fungi communities highlighted changes in composition due to treatments where NT samples were clearly separated from the treatment samples (Figure 2C). The first two components accounted for explaining the $55.8 \%$ of the total variance. Moreover, PERMANOVA tests comparing the different treatment combination showed clear distinctions between them (Table 1). Likewise, the NMDS showed two clusters with NT clearly separated from the samples after treatments (Figure 2D). PERMANOVA test highlighted how treatments affected fungal beta-diversity $(F=7.615, p=$ 0.001). These results suggested that the main factor affecting the bacterial and fungal composition was the time of sampling and that bacterial composition is more responsive to different irrigation amounts than fungal composition.

\subsection{Bacterial and fungal taxa distribution in the Merlot vineyard bulk soil is significantly affected by AMF inoculation, irrigation treatments and time.}

The taxonomic affiliations of the bacterial OTUs core showed vineyard bulk soil hosted 18 phyla, 38 classes, 71 orders, 130 families and 298 genera (Additional file 3). Different samples were dominated by Proteobacteria phylum that accounted, on average, for more than the $35 \%$ in the bacterial communities. Other common phyla were Actinobacteria (ranged between 15.3\% and $23.9 \%$ ), Verrumicrobia (between 8 and 12.2\%), Gemmatimonadetes (between 4.8 and 9.8\%), Firmicutes (between 2.3 and $7.7 \%$ ), Planctomycetes (between 5.6 and 8.8\%), and Chloroflexi (between 1.5 and 2.9\%), as well as the phylum Crenarchaeota belonging to Archaea (ranged between 0 and 16\%) (Figure 3A). 
Irrigation, AMF inoculation and time affected the abundances at phylum levels as subjected the significance of the LMEM conducted (Additional file 4: Table S2A). Thus, FI had increased the proportion of Proteobacteria ( $\mathrm{p} \leq 0.0001)$ while reduced applied water with $\mathrm{HI}$ increased the abundances of Actinobacteria $(p=0.032)$, Chloroflexi $(p=0.019)$ and others $(p \leq 0.0001)$.

The most abundant 12 bacterial classes, which accounted for more than $90 \%$ of the relative abundance in all treatments, are presented in Figure $3 \mathrm{C}$. Irrigation and AMF inoculation treatments affected the proportion of these bacterial classes as highlighted with the significance of the LMEM (Additional file 4: Table S2B). Therefore, we observed increased abundance of Bacilli and decreased abundance of Gemmatimonadetes comparing to the abundances of NT ( $p \leq 0.0001$ for both classes). On the other hand, $\mathrm{Fl}$ and/ or AMF inoculation increased the abundances of Alphaproteobacteria, Betaproteobacteria and Gammaproteobacteria. On the other hand, HINI decreased Alphaproteobacteria and Betaproteobacteria and increased Gammaproteobacteria and Actinobacteria classes. Finally, full irrigation decreased the abundance of Thaumarchaeota and other bacterial classes $(p \leq 0.0001$ and $p=$ 0.003 , respectively, Additional file 4: Table S2B).

Regarding the bulk soil mycobioma different plots showed 4 phyla, 13 classes, 37 orders, 67 families and 121 genera (Additional file 4). The most abundant phyla we found were Basidiomycota, which, on average, accounted for the $50 \%$ of the fungal communities, Ascomycota (ranged between 21.6 and 37.5 \%) and Zygomycota (ranged between 3.3 and $17.6 \%$ ) (Figure 3B).

Relative abundances of the fungal phylum were highly responsive to the treatments as indicated by the LMEM (Additional file 4: Table S3A). Thus, Ascomycota decreased in HINI plots ( $p=0.007)$ while Basidiomycota increased after treatment application in all plots $(\mathrm{p} \leq 0.0001)$. The abundance of Zygomycota phylum increased with the combination of treatments, especially in $\mathrm{Fl}$ and inoculation with AMF ( $p \leq 0.0001$ ) whereas, the abundances of other less abundant phyla decreased (Figure 3C, Additional file 4: Table S3A).

Within fungal classes the most abundant were Agaricomycetes, Dothideomycetes, Eurotiomycetes, Leotiomycetes, Pezizomycetes, Saccharomycetes, Sordariomycetes, and Tremellomycetes, which accounted for the $50 \%$ of the fungal abundance in the NT plots, and between the $62 \%$ and $92 \%$ in the plots after treatment (Figure 3D). However, distribution of fungal classes was strongly affected by treatments (Additional file 4: Table S3B). Non-inoculated plots decreased the abundances of Agaromycetes $(p=0.008)$, Leotimycetes $(p \leq 0.0001)$ and Saccharomycetes $(p=0.001)$ especially in the FI plots. On the other hand, all the treatments increased the abundance of Tremellomycetes in detriment of Sordariomycetes and Dothideomycetes classes. Finally, comparing to NT, plots after treatment application decreased abundances of other fungal classes ( $p \leq 0.0001$, Figure 3D; Additional file 4: Table S3B).

\subsection{Bacterial and fungal clade proportions in the Merlot vineyard after treatments differed due to AMF inoculation and/or irrigation treatments.}


The study of the differences on the median proportions of the microbiome families cladograms due to treatments are presented in the Additional file 1: Figures S4-S10. Within the Proteobacteria phylum (Additional file 1: Figure S4), HI increased the proportion of Oceanospirillaceae and Cellvibrionaceae families. FII increased the Marinicellaceae, Anaeromyxobacteriaceae, Rickettsiaceae and decreased Neisseriaceae. The main changes in Actinobacteria (Additional file 1: Figure S5) were increments of the proportions of Streptoporangiales and Cryptosporangiaceae due to $\mathrm{HI}$, and enhancement of Micromonosporaceae, Acidimicrobiaceae and Actinospicaceae in FII, HINI and HII plots. Firmicutes phylum proportions were highly affected by treatments (Additional file 1: Figure S6). Thus, $\mathrm{HI}$ increased the proportion of members of the family XVII and Erysipelotrichaceae. Fll increased Syntrophomonadaceae and Gracilibacteraceae proportions. Finally, comparing to FINI plots, FII, HII and HINI enhanced Halanaerobiaceae. Within the other less abundant phyla (Additional file 1: Figure S7), the main differences were due to $\mathrm{Hl}$, which, increased families from Chlamydiales and Spirochaetia clades and enhanced the proportions of families belonging to Archaea Kingdom. On the other hand, FII increased the proportion of Deinococcaceae family.

Regarding the effect of different treatments on the proportion of fungal families, members of the Ascomycota phylum were highly affected by treatments (Additional file 1: Figure S8). Fll increased the proportion of Glomerellaceae and Togniniaceae, HII increased Sporormiaceae, Tubeufiaceae and decreased Eremomycetaceae, whereas FI led to increased proportions of Leptosphaeriaceae. Basidiomycota phylum cladogram (Additional file 1: Figure S9) shows that different irrigation treatments affected the Malasseziaceae (increased in $\mathrm{HI}$ ) and Sparassidaceae (increased in FI) families. On the other hand, AMF inoculation increased the proportions of Hydnodontaceae under Fl conditions and Psathyrellaceae and Cortinariaceae under HI conditions. Zygomycota families were not highly affected by treatments (Additional file 1: Figure S10).

\subsection{Edaphic factors barely affected in the bacterial and fungal distribution.}

The SWC decreased with the HI treatment that accounted for a decreased soil evaporation, especially in HII plots, although no interactive effect between factors was evident. The edaphic factors of different plots slightly influenced the bacterial and fungal communities as showed the canonical correspondence analysis (CCA) (Figure 4, Additional file 4: Table S4 and S5).

Table 2. Edaphic factors of Merlot vineyard soil subjected to different irrigation amounts ( $\mathrm{Fl}$, Full Irrigated or $\mathrm{HI}$, Half Irrigated), and AMF inoculation (I, inoculated; or $\mathrm{NI}$, non-inoculated). 


\begin{tabular}{|c|c|c|c|c|c|c|}
\hline & $\begin{array}{l}\text { Soil } \\
\text { pH }\end{array}$ & $\begin{array}{l}\text { Relative } \\
\text { humidity } \\
\text { (RH) }\end{array}$ & $\begin{array}{l}\text { Soil evaporation } \\
\left(\mathrm{mmol} \mathrm{m} \mathrm{m}^{-2} \mathrm{~s}^{-1}\right)\end{array}$ & $\begin{array}{l}\text { Soil } \\
\text { temperature } \\
\left({ }^{\circ} \mathrm{C}\right)\end{array}$ & $\begin{array}{l}\text { SWC } \\
(\mathrm{g} / \mathrm{g})\end{array}$ & $\begin{array}{l}\text { Bulk density } \\
\left(\mathrm{g} / \mathrm{cm}^{3}\right)\end{array}$ \\
\hline $\mathrm{FI}$ & $\begin{array}{l}5.90 \\
\pm \\
0.10\end{array}$ & $17.4 \pm 1.2$ & $0.41 \pm 0.03 \mathbf{a}$ & $39.1 \pm 1.0$ & $\begin{array}{l}0.06 \pm \\
0.01 \mathrm{a}\end{array}$ & $1.11 \pm 0.02$ \\
\hline $\mathrm{HI}$ & $\begin{array}{l}5.74 \\
\pm \\
0.09\end{array}$ & $16.1 \pm 0.5$ & $0.24 \pm 0.02$ b & $39.2 \pm 0.5$ & $\begin{array}{l}0.05 \pm \\
0.01 \mathrm{~b}\end{array}$ & $1.12 \pm 0.01$ \\
\hline $\mathrm{NI}$ & $\begin{array}{l}5.74 \\
\pm \\
0.05\end{array}$ & $16.2 \pm 0.9$ & $0.29 \pm 0.03 \mathbf{b}$ & $39.7 \pm 0.5$ & $\begin{array}{l}0.05 \pm \\
0.01\end{array}$ & $1.11 \pm 0.02$ \\
\hline I & $\begin{array}{l}5.89 \\
\pm \\
0.12\end{array}$ & $17.3 \pm 1.0$ & $0.36 \pm 0.04 \mathbf{a}$ & $38.6 \pm 0.9$ & $\begin{array}{l}0.06 \pm \\
0.01\end{array}$ & $1.11 \pm 0.02$ \\
\hline \multicolumn{7}{|l|}{ LMEM } \\
\hline Irrigation (I) & 0.194 & 0.232 & 0.0001 & 0.862 & 0.029 & 0.591 \\
\hline $\begin{array}{l}\text { AMF } \\
\text { inoculation } \\
\text { (M) }\end{array}$ & 0.155 & 0.267 & 0.017 & 0.199 & 0.094 & 0.935 \\
\hline IXM & 0.267 & 0.780 & 0.413 & 0.645 & 0.331 & 0.264 \\
\hline
\end{tabular}

Values represent means $\pm S E(n=8)$ separated by Kenward-Roger method and Tukey's $p$-value adjustment $(P \leq 0.05)$. Different letters indicate significant differences as affected by treatment application $(\mathrm{Fl}, \mathrm{HI}, \mathrm{NI}$, or I) according to the main factors in the linear mixed-effect model.

However, Figure 4A shows that soil temperature and bulk density, soil evaporation and soil water content correlated with the bacterial beta diversity, and explained the separation between $\mathrm{HI}$ and $\mathrm{FI}$ treatments. This separation was related with the abundances of Nitrososphaera $s p$., Jatrophihabitans $s p$., Actinophytocola sp., Pseudonocardia sp., Geodermatophilus sp., Actinomycetospora sp., Rugosimonospora acidiphila or Micromonospora hermanusense species as shown the correlation with CCA1 (Additional file 4: Table S4C).

On the other hand, the treatments applied did not differ in their fungal composition driven by edaphic factors, and all the treatments clustered together (Figure 4B). However, the soil evaporation and the SWC were negatively correlated with the fungal composition that could be related to the abundances of Penicillium, Aspergillus, Crytococcus and Cladorrhinum members (Additional file 4: Table S5C).

3.5 Co-occurrence patterns in bacterial and fungal communities were affected by time and AMF inoculation and/or irrigation treatments. 
The analyses of the co-occurrence bacterial and fungal networks in the bulk soil of the Merlot vineyard showed different connectivity patterns influenced by time (before and after treatment application; Figure 5, Table 3) and by the different treatments (FINI, FII, HINI and/or HII; Figure 6, Table 4). Before treatment, just $973(0.7 \%)$ of the 141796 pair-wise comparisons yielded statistically significant co-occurrence, comprising 615 positive and 358 negative associations (Table 3). Similarly, after treatment application, from the 135356 pair-wise comparisons, only 1049 were statistically significant with 795 positive and 254 negative co-occurrences, respectively. Thus, although the total number of co-occurrences did not differ between samples before and after treatment application, the latter showed more positive and less negative associations comparing to the pre-treatment samples (Table 3, Figure 5). This shift was likely influenced by the enhancement of the positive associations between bacteria species and the diminution of the negative fungal associations in soil samples after treatment application (Table 3).

Table 3: Degree of connection for bacterial and fungal communities found in Merlot vineyard plots before (not treated, NT) and after treatments, different irrigation amounts (Fl, Full Irrigated or HI, Half Irrigated), and AMF inoculation (I, inoculated; or NI, non-inoculated).

\begin{tabular}{|cll|}
\hline & Not treated (NT) & After treatment \\
\hline Positive connections & & \\
\hline Total & 615 & 795 \\
\hline Bac-Bac & 281 & 350 \\
\hline Fun-Fun & 126 & 205 \\
\hline Bac-Fun & 208 & 240 \\
\hline Negative connections & & \\
\hline Total & 358 & 254 \\
\hline Bac-Bac & 93 & 85 \\
\hline Fun-Fun & 85 & 18 \\
\hline Bac-Fun & 180 & 151 \\
\hline Total connections & & 1049 \\
\hline Total & 973 & 435 \\
\hline Bac-Bac & 374 & 223 \\
\hline Fun-Fun & 211 & 391 \\
\hline Bac-Fun & 388 & 135356 \\
\hline Total analyzed pairs & 141796 & 0.8 \\
\hline Percentage of non-random & 0.7 & \\
\hline
\end{tabular}


We also conducted co-occurrence analyses to assess the effect of AMF inoculation and irrigation treatments (Figure 6, Table 4). In the FI plots, 229 co-occurrences, were found to be statistically significant, majority of them were bacterial associations, bacteria-fungi associations and less frequent the associations between fungal species. The $57 \%$ (130) of the co-occurrences in FI plots were positive while the $43 \%$ were negative $(99$, Table 4$)$. Figure $6 \mathrm{~A}$ showed that the significant negative co-occurrences happened between bacterial and fungal species separately. For instance, Chloroflexi and Acidobacteria with Proteobacteria or connections between Ascomycota species. The positive connectivity found in FI plots was mainly explained by associations between bacteria species, such as the links of Bacteroidetes, Firmicutes, Proteobacteria and Actinobacteria species. On the other hand, HI plots had 300 significant associations, the $77 \%$ (231) of the connections between microbial species were positive while the $23 \%$ (69) were negative. In these plots, half of the connections were identified as fungi-bacteria associations. Thus, a great connectivity between species of Acidobacteria or Proteobacteria with Ascomycota and Basidiomycota members was found (Figure 6B).

Table 4: Degree of connection for bacterial and fungal communities in Merlot vineyard soils subjected to different irrigation amounts (FI, Full Irrigated or $\mathrm{HI}$, Half Irrigated) or AMF inoculation (I, inoculated; or $\mathrm{NI}$, non-inoculated). 


\begin{tabular}{|lllll|}
\hline & FI & HI & NI & I \\
\hline Positive connections & & & & \\
\hline Total & 130 & 231 & 176 & 196 \\
\hline Bac-Bac & 72 & 52 & 97 & 66 \\
\hline Fun-Fun & 24 & 81 & 26 & 62 \\
\hline Bac-Fun & 34 & 98 & 53 & 68 \\
\hline Negative connections & & & & \\
\hline Total & 99 & 69 & 95 & 67 \\
\hline Bac-Bac & 30 & 24 & 25 & 28 \\
\hline Fun-Fun & 21 & 0 & 17 & 0 \\
\hline Bac-Fun & 48 & 45 & 53 & 39 \\
\hline Total connections & & & & \\
\hline Total & 229 & 300 & 271 & 263 \\
\hline Bac-Bac & 102 & 76 & 122 & 94 \\
\hline Fun-Fun & 45 & 81 & 43 & 62 \\
\hline Bac-Fun & 82 & 143 & 106 & 107 \\
\hline Total analyzed pairs & 120299 & 128975 & 128097 & 126817 \\
\hline Percentage of non-random & 0.2 & 0.8 & 0.2 & 0.2 \\
\hline
\end{tabular}

Regarding NI plots, 271 significant association were found with 176 being positive and 95 being negative (Table 4). Majority of the associations were between bacteria species (about $45 \%$, Table 4 ) or between bacteria and fungi (39\%). Negative associations were more frequent between species belonging to Acidobacteria, Proteobacteria, Bacteroidetes or Chloroflexi phyla whereas positive associations were found between Actinobacteria, Verrumicrobia, Proteobacteria and other bacteria phyla (Figure 6C). Moreover, a great degree of connectivity (either positive or negative) between Acidobacteria and Proteobacteria with Ascomycota, Basidiomycota and Zygomycota species was highlighted. Finally, inoculated plots showed 263 significant associations with the $75 \%$ being positive co-occurrences (196) and the $25 \%$ negative $(67$, Table 4$)$. Again, the majority of connections were within bacteria species or between bacteria and fungi species. After inoculation, associations between fungi and bacteria were found, such as Proteobacteria with Ascomycota or Verrumicrobia with Basidiomycota. Contrarily, the connectivity between fungal species was very low. It is noteworthy the increase of the negative 
connectivity network of Acidobacteria species, especially with Verrumicrobia and bacteria belonging to other clades, and Actinobacteria phyla with fungal and bacterial species (Figure 6D).

\section{Discussion}

Fungi and bacteria kingdoms are the main components of soil microbiome. In recent years, the interest in vineyard soil microbiome has further grown due to its importance on the 'terroir' affecting the grapevine health, yield, and quality $[4,6,23]$, as well as the typicity of wines [8, 9, 11]. Bokulich et al. [9] demonstrated that the microbial activity, combined with the abiotic and biotic factors, contributes to characterize wine 'terroir'. Furthermore, several studies have reported that vineyard soil microbiome is related to the grapevine and berry microbiome $[4,8,9]$ affecting the wine composition [38] and fermentation $[6,9,39,40]$.

Regarding the effect of management practices on vineyard microbial communities, previous studies have often been limited to the examination of different soils characterized by geography [13,41,42], vineyard management $[13,43,44]$, rootstocks [10,27,45], and rotundone zones (spatial variability of soil water supply affecting secondary metabolites) [38]. Our aim, however, was to expand the understanding of how the management practices affect the microbiome associated to replanted grapevines by studying them in a short period in a new vineyard. To the best of our knowledge, this is the first study dealing with the effect of the AMF inoculation and water availability on the microbial composition of vineyard soils. However, findings presented here require further studies with for instance, a greater number of locations with different climatic conditions and vineyard ages given the variability in rhizosphere microbiomes between locations $[13,41,42]$ and between young and mature grapevines $[41,46,47]$.

\subsection{Differential responses of bacterial and fungi composition to AMF inoculation and irrigation treatments.}

The bacterial core, meaning the number of shared species between different treatments, was higher than the fungal core. According to Coller et al. [13] majority of the bacterial OTUs were present in a small number of samples, whereas a small number of OTUs was shared by all samples, showing a high degree of variability across samples.

The analysis of alpha and beta diversities indicated that both AMF inoculation and irrigation treatments influenced the bulk soil microbial communities. However, fungal and bacterial communities responded differently to the irrigation treatments and the AMF inoculation according to the alpha diversity indices presented in this study, where bacteria species richness was not affected by the treatments in contrast to previous studies [13]. However, Shannon index increased in half irrigated plots. Likewise, Willing et al. [48] reported that increasing water-availability across the coastal redwood range decreased bacterial species richness estimated as Shannon index, suggesting that the turnover in bacterial communities was most likely to be driven by species loss with increasing water-availability instead of species replacement. Under our experimental conditions, bacterial alpha diversity was higher than fungal alpha diversity in 
accordance with Liang et al. [49], who suggested that bacteria probably played roles that were more pivotal than fungi in vineyard soils.

On the other hand, fungal richness decreased after treatment application, especially in the plots subjected to full irrigation. Conversely, Alonso et al. [40] found that fungal population appeared to be more stable (compared to bacterial communities) in terms of alpha diversity, while Coller et al. [13] did not report significant effect of different land use on core soil mycobiome. However, our results are corroborated by Zhang et al. [50] who reported that after 36 years of irrigation or straw cover management of a wheat production area in China, fungal alpha diversity indices were decreased with irrigation comparing to straw cover treatment whereas no differences were found in bacterial alpha diversity indices. It is known that the composition of fungal communities in soils are very responsive to plant root-exudate composition, which would vary with the plant phenology, especially during flowering and ripening [51] and/or responding to environmental conditions [52].

A previous study on onion rhizosphere showed that inoculation with AMF affect microbial composition accounting for increased dissimilarities, especially in bacterial communities due to the introduction of AMF may include their associated bacteria [22]. However, the analysis of beta diversity in our samples partially corroborated this, the weak effect of AMF inoculation we observed suggested that this effect may be diluted with the distance to the plant roots. On the other hand, the alteration of the soil moisture through irrigation practices may influence the abundance of soil microorganisms, their structure and function, which modified the effect of the irrigation program on the vine performance [53]. In this study, the PCoA and the NMDS analyses suggested that the irrigation applied and especially, the time course played a major role in selecting the bacterial component of the microbiome on vineyard bulk soil rather than the AMF inoculation. Regarding fungal diversity, dissimilarities in soil fungi were generally driven by the time course. Thus, the PCoA and NMDS of the Bray Curtis distances showed that the date of sampling was the main source of beta diversity for fungal communities. Therefore, regarding the beta diversity, bacterial communities seemed to be more responsive to treatments and time course than fungal communities were, corroborating previous studies in must microbiome analysis [8]. Accordingly, several studies documented the temporal heterogeneity based on the inter-annual variability in vineyard soils [41, $45,54,55]$, but few of them studied this effect within the same season $[41,56]$. Therefore, microbiome studies should consider the high degree of temporal variability in the experimental design, because sampling the same point in different times can give different results due to the variability of the own microbial community through time [57].

\subsection{Bacterial and fungal composition after AMF inoculation and irrigation treatment application during the season.}

Previous studies had reported that Proteobacteria and Actinobacteria phyla were predominant in vineyard soils covering about the $50 \%$ of the bacterial relative abundances $[27,38,41,44,49,56]$ and our data corroborated these studies suggesting that the bacterial microbiome in the bulk soil is partially conserved. Furthermore, a recent research with a proteomic approach suggested that Proteobacteria, 
Actinobacteria and Firmicutes were the most active phyla in vineyards in protein expression, and were mainly involved in phosphorus and nitrogen rhizosphere metabolism [58] and in the carbon biochemical cycle and their production of secondary metabolites [59]. Under our experimental conditions, Actinobacteria and Chloroflexi phylum increased in $\mathrm{HI}$ plots. Accordingly, it was previously reported Moreno-Espíndola et al. [60] in an organic Milpa farm with different moisture, thus these authors suggested that phylotypes belonging to Actinobacteria and Chloroflexi were enriched in dry conditions because their thick cell walls, filamentous growth and spore formation favor them under dry conditions [61]. In contrast, Vink et al. [56] found little variation in Actinobacteria and Proteobacteria proportions between the different cultivars, the phenological stages and irrigation treatments.

At the class level, Alphaproteobacteria were the dominant class with frequencies about $20 \%$ in all the soil samples, followed by Actinobacteria ( 10\%), Spartobacteria ( 9\%), Gammaproteobacteria ( 9\%) and Thaumarchacota ( 8\%). Several endophytic microorganisms belonging to Actinobacteria, have been reported to control grapevine pathologies given their in vitro antifungal activity against the main fungal pathogens affecting young grapevines in nurseries and thus, decreasing their mortality and infection rates $[46,62]$. On the other hand, under our experimental conditions AMF and/or FI treatments increased proportions of Alphaproteobacteria, Betaproteobacteria and Gammaproteobacteria. Similarly, Ci et al. [63] recently reported that Alphaproteobacteria increased in the peanut (Arachis hypogaea L.) rhizosphere after AMF inoculation.

At the phylum level, Ascomycota and Basidiomycota were the most abundant phyla detected in all samples accounted for about the $50 \%$ before treatment applications and for almost the $80 \%$ of the relative abundance after treatment application. Previous studies also agreed on the most common fungal phyla detected in vineyard soils $[5,13,41,42,47,49]$. Our results showed an increase in Ascomycota and Zygomycota relative abundances after AMF inoculation. Thus, previous studies reported that Ascomycetes are responding to small scale changes in soil chemistry, water and resource concentrations rather than geomorphic land system classifiers [64]. Furthermore, Zhang et al. [65] demonstrated that Ascomycota proportions in the rhizosphere were enhanced after the inoculation of alfalfa with an endophyte (Trichoderma harzianum).

Soil living fungi provide several ecosystem services such as the biological control, ecosystem regulation, organic matter decomposition and/or composite transformation, among others, with Ascomycota participating in the decomposition of organic matter [66]. On the other hand, members of Zygomycota phylum have been pointed out as suppressors of soil plant pathogens [67].

\subsection{Irrigation treatments and AMF inoculation shifted microbial communities but not through changes in soil edaphic factors.}

Our results showed that AMF inoculation and irrigation treatments slightly affected physicochemical properties of soils, where only soil water evaporation and soil water content were enhanced in FI plots. These results are in agreement with previous studies that did not find changes in $\mathrm{pH}$ and soil moisture in spite of the intra-vineyard variated zones and soil depths [49]. The soil physicochemical properties and 
moisture content have been identified as major factors shaping the spatial scaling of the grapevine rhizosphere microbiome in many previous studies $[4,11,53]$. Our results partially supported this according to the two CCA conducted. We found that edaphic factors barely correlated with bacterial and fungal community dissimilarities. Contrarily, Coller et al. [13] reported that soil usage has a stronger effect on the fungal component of the soil microbiota than on bacterial component.

Within Proteobacteria phylum, the main observed changes in composition were driven by irrigation treatments. Thus, $\mathrm{HI}$ soils incremented members of Oceanospirillaceae and Cellvibrionaceae families. This is in agreement with a recent study that proposed that new species of Oceanospirillaceae family may help rice to overcome the saline stress and promote plant growth by increased the ACC activity [68]. In addition, the increment of Cellvibrionaceae may be related to a potential saline-alkaline stress reported in a previous study dealing with Cadmium-contaminated soils [69]. The $\mathrm{HI}$ treatment enhanced the presence of genus belonging to Actinobacteria which are known to be endophytes related to impair the decline of young grapevines caused by the fungal trunk pathogen infection through the root system in nurseries [70]. This enhancement was mainly due to increased presence of genus Fodinicola, which was recently suggested as a promising source of novel secondary metabolites for enhancing plant growth [71]. In addition, AMF and HI enhanced the presence of Micromonosporaceae, which was related with the plant health in $\mathrm{N}$-fixing plants and enhancing the growth of several host plants under controlled conditions [72]. In these treatments the presence of members belonging to Actinospicaceae family also increased, likely improving the antifungal activity, siderophore production and phosphate solubilization activity [73].

Candidate division TM7 increased after AMF inoculation or with half irrigation. This clade was suggested as a key biomarker of the resistance against wilt disease in tobacco [74] and after Verticillium inoculation in olive [75]. Thus, those studies showed decreases in its relative abundance in infected plants. Therefore, increased presence of this clade could be associated with a better soil quality.

With regard to fungi, Ascomycota composition was highly affected by AMF inoculation and irrigation treatments. Thus, AMF inoculation under FI conditions increased the proportion of plant pathogens such as Phaeoacremonium (Togniciaceae family) which has been related with Esca disease in grapevines [76] and Glomerella tucumanensis (Glomerellaceae family) responsible of the red root rot disease in sugarcane affecting sugar production and productivity [77]. However, under HI conditions, AMF inoculation increased the proportion of Preussia (Sporormiaceae family), an endophytic fungus isolated from Vitis labrusca L. leaves without in vitro activity against the pathogen Fusarium oxysporum [78]. The FI treatment also increased the proportion of Coniothyrium (Leptosphaeriaceae) which was subjected as a potential biocontrol of Sclerotinia lettuce drop [79].

Within Basidiomycetes, AMF inoculation increased the presence of Psathyrellaceae family whose members are known to be endophytes which may contribute to grapevine growth and productivity [80] and are very abundant in organic farmlands [81]. Inoculated soils also increased the presence of 
Hydonodontaceae, which has been subjected to be a potential bioindicator given the negative relationship with the ginseng (Panax notoginseng) mortality in a continuous cropping system [82].

\subsection{Time and management practices affect bacterial and fungal co-occurrence networks.}

Network analysis of microbial community is a useful tool that allows assessing the community structure and the potential interactions between its members [83]. It is worth mentioning that co-occurrence network visualization showed the correlative relationships between taxa, including the true ecological interactions (i.e. mutualism) but also nonrandom processes (i.e. niche-overlap), and therefore, they do not necessarily reflect direct interactions between species [15]. Nevertheless, they were still convenient for exploring abundance patterns in complex microbial communities and evaluating effects of different managements.

Under our experimental conditions, the co-occurrence analyses revealed majority of 'random' association between species. However, there were two possible categories of random associations. Accordingly, two species can be classified as a random association because the statistical power is not sufficient, although the difference between observed and expected co-occurrence is substantial, or because the association between two species is truly random, with similar observed and expected co-occurrences [36]. Further studies would need to distinguish the associations classified as random and to difference between truly random or positive by increasing the number of sampled sites. Comparing the networks before and after treatment, we have shown evidence that the number of positive associations between microbes increased. In other words, the observed co-occurrence of two species was greater than the expected and species tended to occur together at more locations than expected when they are randomly distributed relative to the other species. Contrarily, negative associations were decreased, namely, two species co-occurred at fewer locations than expected given that they were randomly distributed. This led to a more complex soil microbial community network along the season, which has been suggested to benefit plants [84]. Indeed, Layeghifard et al. [84] suggested that the functional capacity of microbiome is not equal to the sum of its individual components, given the interaction between microbial species and the formation of complex networks that significantly influence ecological processes and host adaptations [85]. Our results corroborated this hypothesis although species richness decreased along time, especially in the fungal community, the number of positive associations increased after treatments accounted for a $24 \%$ for connections between bacteria species, a $62 \%$ between fungal species and a $15 \%$ for fungi-bacteria associations.

The comparison of treatments showed different patterns of connectivity within after treatment samples. Thus, our findings suggest that compared to $\mathrm{Fl}, \mathrm{HI}$ increased the connectivity between species, increasing the number of positive co-occurrences and promoting the associations between fungal species and fungal and bacterial species. Accordingly, de Vries et al. [16] found that soil bacterial networks were less stable under drought than fungal networks. Thus, these authors reported that drought stress promoted destabilizing properties of bacterial co-occurrence networks via changes in vegetation composition and resultant reductions in soil moisture. 
On the other hand, the analysis of inoculated and not inoculated plots showed similar degree of connectivity. However, the inoculation with AMF promoted the positive associations in detriment of the negative associations between species. Previous studies reported that inoculation of plants with plantgrowth promoting bacteria led to more complex and compact associations in their associated microbiome. Accordingly, previous research showed that inoculation of Camelia sinensis with a microbial consortium strengthened the connection between the bacteria, indicating a greater stability of the community comparted to the control and promotion of cooperation after inoculation [20]. Similarly, in soybean rhizosphere, it was reported that the inoculation with Rhizobium, a natural $\mathrm{N}$-fixing bacteria symbiont of leguminous plants, led to increased number of connections between fungal species [21]. Consequently, a previous study reported that the soil of AMF inoculated onions showed that AMF cooccurred with several indigenous bacteria and fungi, suggesting that inoculated AMF fungi may recruit specific taxa to confer a better plant performance [22]. These changes in network connectivity were likely explained by the modification of plant signaling molecules, hormones and exudate composition that take place with the mycorrhizal symbiosis and that are known to modify the soil characteristics [17]. Furthermore, although it was hypothesized that the plant symbionts may modify the environment and therefore the microbial structure [17], to the best of our knowledge, little experimental evidence is currently available. Therefore, our work gives some insights about the effects that cultural practices may have on the microbial community structure and connectivity.

\section{Conclusion}

We conducted this experiment to assess whether the vineyard soil microbiome was altered by different management practices such as use of biostimulants (AMF inoculation vs. non-inoculated) and/or irrigation management (full irrigated vs. half irrigated). Our results indicated that bacterial and fungal communities in vineyard soils are shaped by both time course and soil management (i.e. use of biostimulants or irrigation) and more importantly, there was an interactive effect observed among these factors. However, different sensibilities within microbes were found when analyzing alpha and beta diversities. Thus, alpha diversity was more responsive to treatments in the fungal communities whereas changes on beta diversity were mainly recorded in the bacterial communities. Microbial network analyses suggested that the bacterial associations were weaker than the fungal ones under half irrigation and that the inoculation with AMF led to the increase of positive associations between vineyard soil-living microbes. Altogether, our results highlighted the necessity of more studies leading with the effect of management practices and especially the addition of AMF to cropping systems may have to the soil microbiota in order to strengthen beneficial microbial networks and consequently, achieve a better soil quality that improves crop performance.

\section{List Of Abbreviations}

AMF: Arbuscular mycorrhizal fungi; NT: Not treated plots (plots before treatment); FINI: vineyard plots full irrigated and non-inoculated; FIl: vineyard plots full irrigated and inoculated with AMF; HINI: vineyard plots 
half irrigated and non-inoculated; HII: vineyard plots half irrigated and inoculated with AMF; OTU:

Operational taxonomic unit; LMEM: Linear mixed-Effect models; PCoA: Principal coordinates analysis; PERMANOVA: Permutational multivariate analysis of variance; NMDS: Nonmetric Multidimensional Scaling; CCA: Canonical Correspondence Analysis; SWC: Soil water content.

\section{Declarations}

\section{Ethics approval and consent to participate}

Not applicable.

\section{Consent for publication}

Not applicable.

\section{Availability of data and materials}

The dataset supporting the conclusions of this article is included within the article (Additional file 3 ). We also provided the R script for data analysis and al necessary input files as Additional file 2.

\section{Competing interests}

The authors declare that they have no competing interests.

\section{Funding}

Post-doctoral student fellowships were provided to NT and RY from Department of Viticulture and Enology at University of California Davis during the execution of this study.

\section{Author's contributions}

NT conceived the study, performed the sample collection, data analysis and wrote the first version of the manuscript. RY contribute to sample collection and manuscript redaction. SKK acquired the funding; contribute to the experimental design, and manuscript redaction. All authors critically reviewed and edited the manuscript and approved its publication.

\section{Acknowledgements}

The authors thank Biome Makers for the microbiome analyses.

\section{References}

1. Compant S, Samad A, Faist H, Sessitsch A. A review on the plant microbiome: Ecology, functions, and emerging trends in microbial application. J Adv Res. 2019;19:29-37. DOI: 10.1016/j.jare.2019.03.004. 
2. Karimi B, Cahurel J-Y, Gontier L, Charlier L, Chovelon M, Mahé H, Ranjard L. A meta-analysis of the ecotoxicological impact of viticultural practices on soil biodiversity. Environ Chem Lett. 2020;18:1947-1966. DOI: 10.1007/s10311-020-01050-5

3. Lazcano C, Decock C, Wilson SG. Defining and Managing for Healthy Vineyard Soils, Intersections with the Concept of Terroir. Front Environ Sci. 2020;8:68. DOI: 10.3389/fenvs.2020.00068.

4. Zarraonaindia I, Owens SM, Weisenhorn P, West K, Hampton-Marcell J, Lax S, Bokulich NA, Mills DA, Martin G, Taghavi S, van der Lelie D, Gilbert JA. The soil microbiome influences grapevine-associated microbiota. mBio 2015;6:e02527-14. DOI:10.1128/mBio.02527-14.

5. Knight SJ, Karona O, Goddarda, MR. Small scale fungal community differentiation in a vineyard system. Food Microbiol. 2020;87:103358. DOI: 10.1016/j.fm.2019.103358

6. Mezzasalma V, Sandionigi A, Bruni I, Bruno A, Lovicu G, Casiraghi M, et al. Grape microbiome as a reliable and persistent signature of field origin and environmental conditions in Cannonau wine production. PLoS ONE. 2017;12:e0184615. DOI: 10.1371/journal. pone.0184615

7. van Leeuwen C, Roby JP, de Rességuier L. Soil-related terroir factors: a review. OENO One. 2018;52:173-188. DOI: 10.20870/oeno-one.2018.52.2.2208.

8. Bokulich NA, Thorngated JH, Richardsone PM, Mills DA. Microbial biogeography of wine grapes is conditioned by cultivar, vintage, and climate. PNAS. 2014;111:E139-E148 DOI: 10.1073/pnas. 1317377110

9. Bokulich NA, Collins TS, Masarweh C, Allen G, Heymann H, Ebeler SE, Mills DA. Associations among wine grape microbiome, metabolome, and fermentation behavior suggest microbial contribution to regional wine characteristics. MBio. 2016;7:e00631-16. DOI: 10.1128/mBio.00631-16.

10. Burns KN, Bokulich NA, Cantu D, Greenhut RF, Kluepfel DA, O'Geen AT, Strauss SL, Steenwerth KL. Vineyard soil bacterial diversity and composition revealed by 16S rRNA genes: Differentiation by vineyard management. Soil Biol Biochem. 2016;103:337-348. DOI: 10.1016/j.soilbio.2016.09.007

11. Burns KN, Kluepfel DA, Strauss SL, Bokulich NA, Cantu D, Steenwerth KL. Vineyard soil bacterial diversity and composition revealed by $16 \mathrm{~S}$ rRNA genes: Differentiation by geographic features. Soil Biol Biochem. 2015;91:232-247. DOI: 10.1016/j.soilbio.2015.09.002.

12. Likar M, Stres B, Rusjan D, Potisek M, Regvar M. Ecological and conventional viticulture gives rise to distinct fungal and bacterial microbial communities in vineyard soils. Appl Soil Ecol. 2017;113:86-95. DOI: 10.1016/j.apsoil.2017.02.007

13. Coller E, Cestaro A, Zanzotti R, Bertoldi D, Pindo M, Larger S, et al. Microbiome of vineyard soils is shaped by geography and management. Microbiome 2019;7:140. DOI: 10.1186/s40168-019-0758-7

14. Rienth M, Scholasch T. State-of-the-art of tools and methods to assess vine water status. OENO One 2019;4:619-637. DOI:10.20870/oeno-one.2019.53.4.2403

15. Hartman K, van der Heigden MGA, Wittwer RA, Banerjee S, Walser J-C, Schlaeppi K. Cropping practices manipulate abundance patterns of root and soil microbiome members paving the way to smart farming. Microbiome. 2018;6:14. DOI: 10.1186/s40168-017-0389-9. 
16. de Vries FT, Griffiths RI, Bailey M, Craig H, Girlanda M, Gweon HS, et al. Soil bacterial networks are less stable under drought than fungal networks. Nat Commun. 2018;9:3033. DOI: 10.1038/s41467018-05516-7

17. Uroz S, Courty PE, Oger P. Plant Symbionts Are Engineers of the Plant-Associated Microbiome. Trends Plant Sci. 2019;24:905-916. DOI: 10.1016/j.tplants.2019.06.008

18. Finkel OM, Castrillo G, Herrera Paredes S, Salas Gonzalez I, Dangl JL. Understanding and exploiting plant beneficial microbes. Curr Opin Plant Biol. 2017;38:155-163. DOI: 10.1016/j.pbi.2017.04.018.

19. Del Buono D. Can biostimulants be used to mitigate the effect of anthropogenic climate change on agriculture? It is time to respond. Sci Total Environ. 2021;751:141763. DOI:

10.1016/j.scitotenv.2020.141763.

20. Shang J, Liu B. Application of a microbial consortium improves the growth of Camellia sinensis and influences the indigenous rhizosphere bacterial communities. J Appl Microbiol. 2020.

DOI:10.1111/jam.14927

21. Xu H, Yang Y, Tian Y, Xu R, Zhong Y, Liao H. Rhizobium Inoculation Drives the Shifting of Rhizosphere Fungal Community in a Host Genotype Dependent Manner. Front Microbiol. 2020;10:3135. DOI: 10.3389/fmicb.2019.03135.

22. Akyol TY, Niwa R, Hirakawa H, Maruyama H, Sato T, Suzuki T, Fukunaga A, Sato T, Yoshida S, Tawaraya K, Saito M, Ezawa T, Sato S. Impact of Introduction of Arbuscular Mycorrhizal Fungi on the Root Microbial Community in Agricultural Fields. Microbes Environ. 2019;34:23-32. DOI:10.1264/jsme2.ME18109

23. Belda I, Zarraonaindia I, Perisin M, Palacios A, Acedo A. From Vineyard Soil to Wine Fermentation: Microbiome Approximations to Explain the "terroir" Concept. Front Microbiol. 2017;8:821. DOI: 10.3389/fmicb.2017.00821

24. Belda I, Palacios A, Fresno J, Ortega H, Acedo A. WineSeq®: A new tool for the study of the functional biodiversity of soils, and its use as a biomarker and guide for vitiviniculture practices. BIO Web of Conferences. 2017;9:01012. DOI: 10.1051/bioconf/20170901012.

25. Rognes T, Flouri T, Nichols B, Quince C, Mahé F. VSEARCH: a versatile open source tool for metagenomics. PeerJ. 2016;4:e2584. DOI: 10.7717/peerj.2584

26. Chen H. Package 'VennDiagram'. Generate High-Resolution Venn and Euler Plots. Version 1.6.20. 2018

27. Marasco R, Rolli E, Fusi M, Michoud G, Daffonchio D. Grapevine rootstocks shape underground bacterial microbiome and networking but not potential functionality. Microbiome. 2018;6:3. DOI: 10.1186/s40168-017-0391-2

28. Bates, D., Maechler, M., Bolker, B., Walker, S., Christensen, R. H. B., Singmann, H., et al. Package 'Ime4'. Linear Mixed-Effects Models using ‘Eigen’ and S4. 1.1-25. Madison, WI: R Project. 2020.

29. Bates, D., Maechler, M., Bolker, B., and Walker, S. Fitting linear mixed effects models usinglme4. J Stat Softw 2015;67:1-48. DOI: 10.18637/jss.v067.i01 
30. Kuznetsova A, Brockhoff PB, Christensen RHB, Jensen SP. Package 'ImerTest'. Tests in Linear Mixed Effects Models. Version 3.1-3. Denmark: R Project. 2020.

31. Lenth R. Package 'Ismeans'. Least-Squares Means. Version 2.30-0. Ames, IA: R Project. 2018.

32. Oksanen J, Blanchet FG, Friendly M, Kindt R, Legendre P, McGlinn D, Minchin PR, O'Hara RB, Simpson GL, Solymos P, Stevens MHH, Szoecs E, Wagner H. Package 'Vegan'. Community Ecology Package. Version 2.5-6. 2019.

33. Foster Z, Grunwald N, Gilmore R. Package 'Metacoder'. Tools for Parsing, Manipulating, and Graphing Taxonomic Abundance Data. Version 0.3.4. 2020.

34. Roberts DW. Package 'labdsv'. Ordination and Multivariate Analysis for Ecology. Version 2.0-1. 2019.

35. Griffith DM, Veech JA, Marsh CJ. Package 'cooccur'. Probabilistic Species Co-Occurrence Analysis in R. Version 1.3. 2016.

36. Veech JA. A probabilistic model for analysing species co-occurrence. Global Ecol Biogeogr. 2013;22:252-260. DOI: 10.1111/j.1466-8238.2012.00789.x

37. Almende BV, Benoit T, Titouan R. Package 'visNetwork'. Network visualization using 'vis.js' Library. Version 2.0.9. 2019.

38. Gupta VVSR, Bramley RGV, Greenfield P, Yu J, Herderich MJ. Vineyard Soil Microbiome Composition Related to Rotundone Concentration in Australian Cool Climate 'Peppery' Shiraz Grapes. Front Microbiol. 2019;10:1607. DOI: 10.3389/fmicb.2019.01607

39. Grangeteau C, Roullier-Gall C, Rousseaux S, Gougeon RD, Schmitt-Kopplin P, Alexandre H, et al. Wine microbiology is driven by vineyard and winery anthropogenic factors. Microb Biotechnol. 2017;10:354-370. DOI:10.1111/1751-7915.12428

40. Alonso A, de Celis M, Ruiz J, Vicente J, Navascués E, Acedo A, et al. Looking at the Origin: Some Insights into the General and Fermentative Microbiota of Vineyard Soils. Fermentation. 2019;5:78. DOI:10.3390/fermentation5030078

41. Berlanas C, Berbegal M, Elena G, Laidani M, Cibriain JF, Sagües A, Gramaje D. The Fungal and Bacterial Rhizosphere Microbiome Associated With Grapevine Rootstock Genotypes in Mature and Young Vineyards. Front Microbiol. 2019;10:1142. DOI: 10.3389/fmicb.2019.01142.

42. Oyuela Aguilar M, Gobbi A, Browne PD, Ellegaard-Jensen L, Hansen LH, Semorile L, et al. Influence of vintage, geographic location and cultivar on the structure of microbial communities associated with the grapevine Rhizosphere in vineyards of San Juan Province, Argentina. PLoS ONE. 2020;15: e0243848. DOI: 10.1371/journal.pone.0243848.

43. Ortiz-Álvarez R, Ortega-Arranz H, Vicente J. Ontiveros VJ, Ravarani C, Acedo A, Belda I. Emergent properties in microbiome networks reveal the anthropogenic disturbance of farming practices in vineyard soil fungal communities. bioRxiv 2020;983650. DOI: 10.1101/2020.03.12.983650

44. Novello G, Gamalero E, Bona E, Boatti L, Mignone F, Massa N, Cesaro P, Lingua G, Berta G. The Rhizosphere Bacterial Microbiota of Vitis vinifera Pinot Noir in an Integrated Pest Management Vineyard. Front Microbiol. 2017;8:1528. DOI: 10.3389/fmicb.2017.01528 
45. Canfora L, Vendramin E, Felicia B, Tarricone L, Florio A, Benedetti A. Vineyard microbiome variations during different fertilisation practices revealed by 16s rRNA gene sequencing. Appl Soil Ecol. 2018;125:71-80. DOI: 10.1016/j.apsoil.2017.12.019.

46. Andreolli M, Lampis S, Zapparoli G, Angelini E, Vallini G. Diversity of bacterial endophytes in 3 and 15 year-old grapevines of Vitis vinifera Corvina and their potential for plant growth promotion and phytopathogen control. Microbiol Res. 2016;183: 42-52. DOI: 10.1016/j.micres.2015.11.009

47. Manici LM, Saccà ML, Caputo F, Zanzotto A, Gardiman M, Fila G. Long- term grapevine cultivation and agro-environment affect rhizosphere microbiome rather than plant age. Appl Soil Ecol. 2017;119:214-225. DOI: 10.1016/j.apsoil.2017.06.027.

48. Willing CE, Pierroz G, Devin Coleman-Derr D, Dawson TE. The generalizability of water-deficit on bacterial community composition; Site-specific water-availability predicts the bacterial community associated with coast redwood roots. Mol Ecol. 2020;29:4721-4734. DOI: 10.1111/mec.15666

49. Liang H, Wang $X$, Yan J, Luo L. Characterizing the Intra-Vineyard Variation of Soil Bacterial and Fungal Communities. Front Microbiol. 2019;10:1239. DOI: 10.3389/fmicb.2019.01239

50. Zhang R, Gu J, Wang X. Responses of soil bacteria and fungi after 36 years fertilizer, straw cover and irrigation management practices in northwest China. Soil Use Manage. 2020;00:1-12. DOI: $10.1111 /$ sum. 12671.

51. Garcia, J. A. L., Barbas, C., Probanza, A., Barrientos, M. L. \& Manero, F. J. G. Low molecular weight organic acids and fatty acids in root exudates of two Lupinus cultivars at flowering and fruiting stages. Phytochem Anal. 2001;12:305-311.

52. Goss-Souza D, Mendes LW, Borges CD, Baretta D, Tsai SM, Rodrigues JLM. Soil microbial community dynamics and assembly under long-term land use change. FEMS Microbiol Ecol. 2017;93. DOI:1093/femsec/fix109.

53. Holland TC, Bowen P, Bogdanoff C, Hart MM. How distinct are arbuscular mycorrhizal fungal communities associating with grapevines? Biol Fertil Soils 2014;50:667-674. DOI: 10.1007/s00374013-0887-2

54. Chou M-Y, Heuvel JV, Bell TH, Panke-Buisse K. Kao-Knifn J. Vineyard under-vine floor management alters soil microbial composition, while the fruit microbiome shows no corresponding shifts. Sci Rep. 2018;8:11039. DOI: 10.1038/s41598-018-29346-1.

55. Simoni S, Gagnarli E, Goggioli D, Guidi S, Tarchi T, D’Avino L, Giffard B. Impact Of Agricultural Management On Communities Of Oribatida, Gamasina And Collembola In Italian And French Vineyards. EQA. 2018;31:27-32. DOI: 10.6092/issn.2281-4485/7902

56. Vink SN, Chrysargyris A, Tzortzakis N, Falcao - Salles J. Bacterial community dynamics varies with soil management and irrigation practices in grapevines (Vitis vinifera). Appl Soil Ecol. 2021;158:103807. DOI: 10.1016/j.apsoil.2020.103807

57. Redford AJ, Fierer N. Bacterial succession on the leaf surface: a novel system for studying successional dynamics. Microb Ecol. 2009;58:189-198. doi: 10.1007/s00248-009-9495-y. 
58. Bona E, Massa N, Novello G, Boatti L, Cesaro P, Todeschini V et al. Metaproteomic characterization of the Vitis vinifera FEMS Microbiol Ecol. 2019;95:fiy204. DOI: 0000-0002-4320-1203.

59. Jenkins SN, Waite IS, Blackburn A, Husband R, Rushton SP, Manning DC, O'Donnell AG. Actinobacterial community dynamics in long term managed grasslands. Antonie Van Leeuwenhoek. 2009;95:319-334. DOI: 10.1007/s10482-009-9317-8.

60. Moreno-Espíndola IP, Ferrara-Guerrero MJ, Luna-Guido ML, Ramírez-Villanueva DA, De LeónLorenzana AS, Gómez-Acata S, González-Terreros E, et al. The Bacterial Community Structure and Microbial Activity in a Traditional Organic Milpa Farming System under Different Soil Moisture Conditions. Front Microbiol. 2018;9:2737. DOI: 10.3389/fmicb.2018.02737.

61. Santos-Medellín C., Edwards J., Liechty Z., Nguyen B., Sundaresan V. Drought stress results in a compartment-specific restructuring of the rice root-associated microbiomes. mBio 2017;8:e00764-17. DOI:10.1128/mBio.00764-17

62. Álvarez-Pérez JM, González-García S, Cobos R, Olego MÁ, Ibañez A, Díez-Galán A, Garzón-Jimeno E, Coque JJR. Use of endophytic and rhizosphere Actinobacteria from grapevine plants to reduce nursery fungal graft infections that lead to young grapevine decline. Appl Environ Microbiol. 2017;83:e01564-17. DOI: 10.1128/AEM.01564-17.

63. Ci D, Tang Z, Di H, Cui L, Zhang G, Li S, Dai L, Qin F, Zhang Z, Yang J, Xu Y. The synergy effect of arbuscular mycorrhizal fungi symbiosis and exogenous calcium on bacterial community composition and growth performance of peanut (Arachis hypogaea) in saline alkali soil. J Microbiol. 2021; 59:51-63. DOI: $10.1007 /$ s12275-021-0317-3

64. Green JL, Holmes AJ, Westoby M, Oliver I, Briscoe D, Dangerfield M, et al. Spatial scaling of microbial eukaryote diversity. Nature 2004;432:747-750. DOI: 10.1038/nature03034

65. Zhang, F., Xu, X., Huo, Y., Xiao, Y. Trichoderma-inoculation and mowing synergistically altered soil available nutrients, rhizosphere chemical compounds and soil microbial community, potentially driving alfalfa growth. Front Microbiol. 2019;3241. DOI: 10.3389/fmicb.2018.03241.

66. Frac M, Hannula SE, Bełka M, Jedryczka M. Fungal Biodiversity and Their Role in Soil Health. Front Microbiol. 2018;9:707. DOI: 10.3389/fmicb.2018.00707

67. Xiong W, Li R, Ren Y, Liu C, Zhao Q, Wu H, Jousset A, Shen Q. Distinct roles for soil fungal and bacterial communities associated with the suppression of vanilla Fusarium wilt disease. Soil Biol Biochem. 2017; 107:198-207. DOI: 10.1016/j.soilbio.2017.01.010.

68. Krishnan R, Lang E, Midh S, Patil PB, Rameshkumar N. Isolation and characterization of a novel 1aminocyclopropane-1-carboxylate (ACC) deaminase producing plant growth promoting marine Gammaproteobacteria from crops grown in brackish environments. Proposal for Pokkaliibacter plantistimulans nov., sp. nov., Balneatrichaceae fam. nov. in the order Oceanospirillales and an emended description of the genus Balneatrix. Syst Appl Microbiol. 2018;41:570-580. DOI: 10.1016/j.syapm.2018.08.003

69. Wang M, Chen S, Chen L, Wang D. Responses of soil microbial communities and their network interactions to saline-alkaline stress in Cd-contaminated soils. Environ Pollut. 2019;252:1609-1621. 
DOI: 10.1016/j.envpol.2019.06.082.

70. Álvarez-Pérez JM, González-García S, Cobos R, Olego MÁ, Ibañez A, Díez-Galán A, Garzón-Jimeno E, Coque JJR. Use of endophytic and rhizosphere actinobacteria from grapevine plants to reduce nursery fungal graft infections that lead to young grapevine decline. Appl Environ Microbiol. 2017;83:e01564-17. DOI: 1128/AEM.01564-17.

71. Pham HTT, Suwannapan W, Koomsiri W, Inahashi Y, Také A, Matsumoto A, Thamchaipenet A. Fodinicola acaciae nov., an Endophytic Actinomycete Isolated from the Roots of Acacia mangium Willd. and Its Genome Analysis. Microorganisms 2020;8: 467. DOI:10.3390/microorganisms8040467

72. Trujillo ME, Riesco R, Benito P, Carro L. Endophytic Actinobacteria and the Interaction of Micromonospora and Nitrogen Fixing Plants. Front Microbiol. 2015;6:1341. DOI: 10.3389/fmicb.2015.01341

73. Poomthongdee N, Duangmal K, Pathom-aree W. Acidophilic actinomycetes from rhizosphere soil: diversity and properties beneficial to plants. J Antibiot. 2015;68:106-114. DOI:10.1038/ja.2014.117;

74. Shen G, Zhang S, Liu X, Jiang Q, Ding W. Soil acidification amendments change the rhizosphere bacterial community of tobacco in a bacterial wilt affected field. Appl Microbiol Biotechnol. 2018;102:9781-9791. DOI: 10.1007/s00253-018-9347-0

75. Fernández-González AJ, Cardoni M, Gómez-Lama Cabanás C, Valverde-Corredor A, Villadas PJ, Fernández-López M, Mercado-Blanco J. Linking belowground microbial network changes to different tolerance level towards Verticillium wilt of olive. Microbiome 2020;8:11. DOI:10.1186/s40168-0200787-2.

76. Essakhi S, Mugnail L, Crous PW, Groenewald JZ, Surico G. Molecular and phenotypic characterisation of novel Phaeoacremonium species isolated from esca diseased grapevines. Persoonia 2008;21:119-134. DOI:10.3767/003158508X374385

77. Viswanathan R, Selvakumar R, Manivannan K, Nithyanantham R, Kaverinathan K. Behaviour of Soil Borne Inoculum of Colletotrichum falcatum in Causing Red Rot in Sugarcane Varieties with Varying Disease Resistance. Sugar Tech. 2020;22:485-497. DOI: 10.1007/s12355-020-00800-7

78. Brum MCP, Araújo WL, Maki CS, Azevedo JL. Endophytic fungi from Vitis labrusca ('Niagara Rosada') and its potential for the biological control of Fusarium oxysporum. 2012 Genet Mol Res. 2012;11:4187-4197. DOI:10.4238/2012.December.6.2

79. Rabeendran N, Jones EE, Moot DJ, Stewart A. Biocontrol of Sclerotinia lettuce drop by Coniothyrium minitans and Trichoderma hamatum. Biol Control 2006;39:352-362.

80. DOI:10.1016/j.biocontrol.2006.06.004

81. Martinez-Diz MP, Andrés-Sodupe M, Bujanda R, Díaz-Losada E, Eichmeier A, Gramaje A. Soil-plant compartments affect fungal microbiome diversity and composition in grapevine. Fungal Ecol. 2019;41:234-244. DOI: 10.1016/j.funeco.2019.07.003.

82. Banerjee S, Walder F, Büchi L, Meyer M, Held AY, Gattinger A, Keller T, Charles R, van der Heijden MGA. Agricultural intensification reduces microbial network complexity and the abundance of keystone taxa in roots. ISME J. 2019;13:1722-1736. DOI: 10.1038/s41396-019-0383-2 
83. Dong L, Xu J, Feng G, Li X, Chen S. Soil bacterial and fungal community dynamics in relation to Panax notoginseng death rate in a continuous cropping system. Sci Rep. 2016;6:31802. DOI: 10.1038/srep31802

84. Zhou, J., Deng, Y., Luo, F., He, Z., Tu, Q., Zhi, X. (2010). Functional molecular ecological networks. MBio. 2010;1:e00169-10. DOI: 10.1128/mBio.00169-10

85. Tao J, Meng D, Qin C, Liu X, Liang Y, Xiao Y, Liu Y, Gu Y, Li J, Yin H. Integrated network analysis reveals the importance of microbial interaction for maize growth. Appl Microbiol Biotechnol. 2018;102:3805-3818. DOI: 10.1007/s00253-018-8837-4.

86. Layeghifard M, Hwang DM, Guttman DS. Disentangling interactions in the microbiome: a network perspective. Trends Microbiol. 2017;25:217-228. DOI: 10.1016/j.tim.2016.11.008.

\section{Figures}



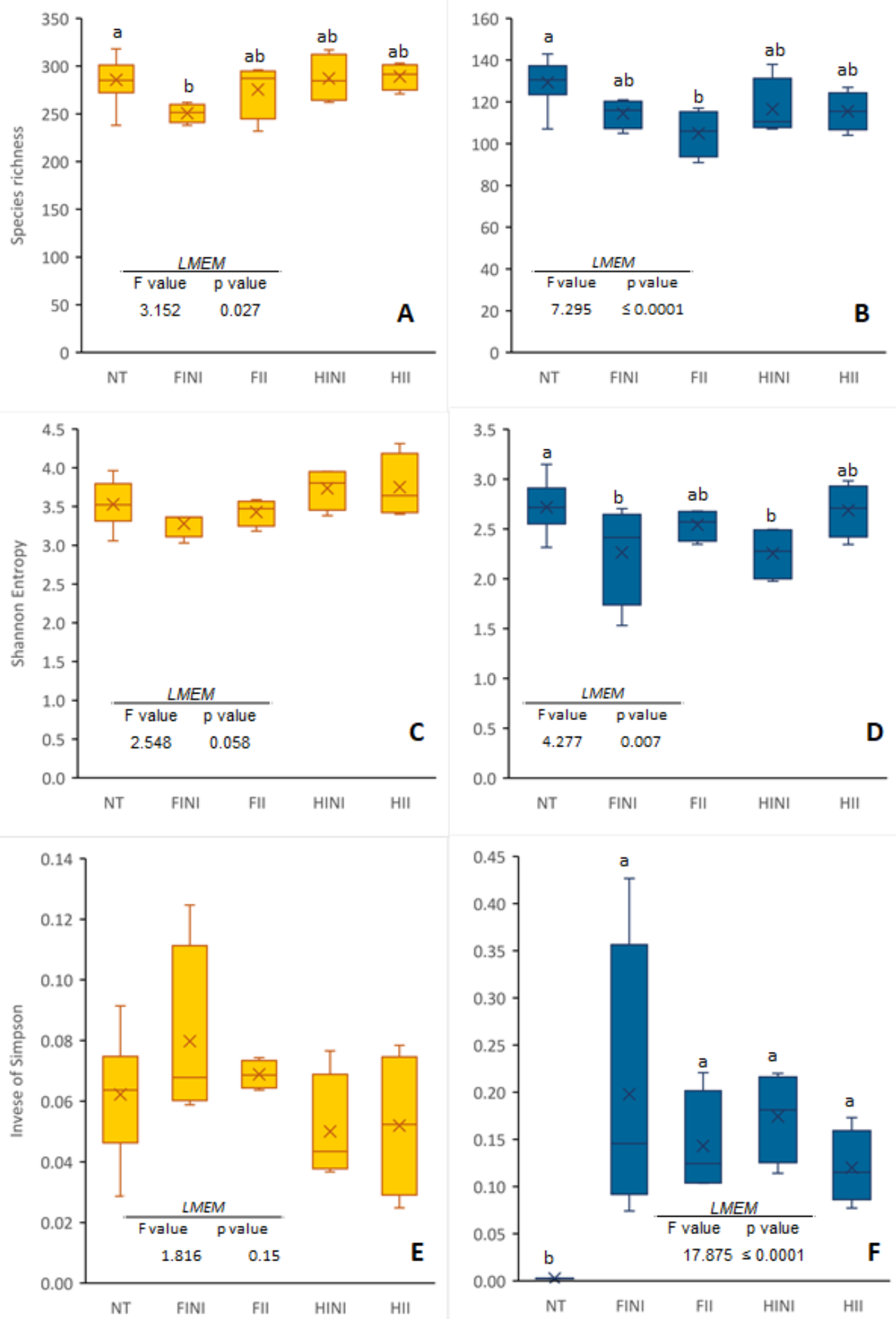

Figure 1

Species richness $(A, B)$, Shannon diversity $(C, D)$ and inverse of Simpson $(E, F)$ indices of bacterial and fungal communities found in plots before (NT) and after treatments, different irrigation amounts (FI, Full Irrigated or $\mathrm{HI}$, Half Irrigated), and AMF inoculation (I, inoculated; or NI, non-inoculated). Values represent means \pm SE $(n=4-16)$ separated by Kenward-Roger method and Tukey's $p$-value adjustment $(P \leq 0.05)$. 
Different letters indicate significant differences as affected by NT, or treatment application (FINI, FII, HINI or HII) according to the linear mixed-effect model.
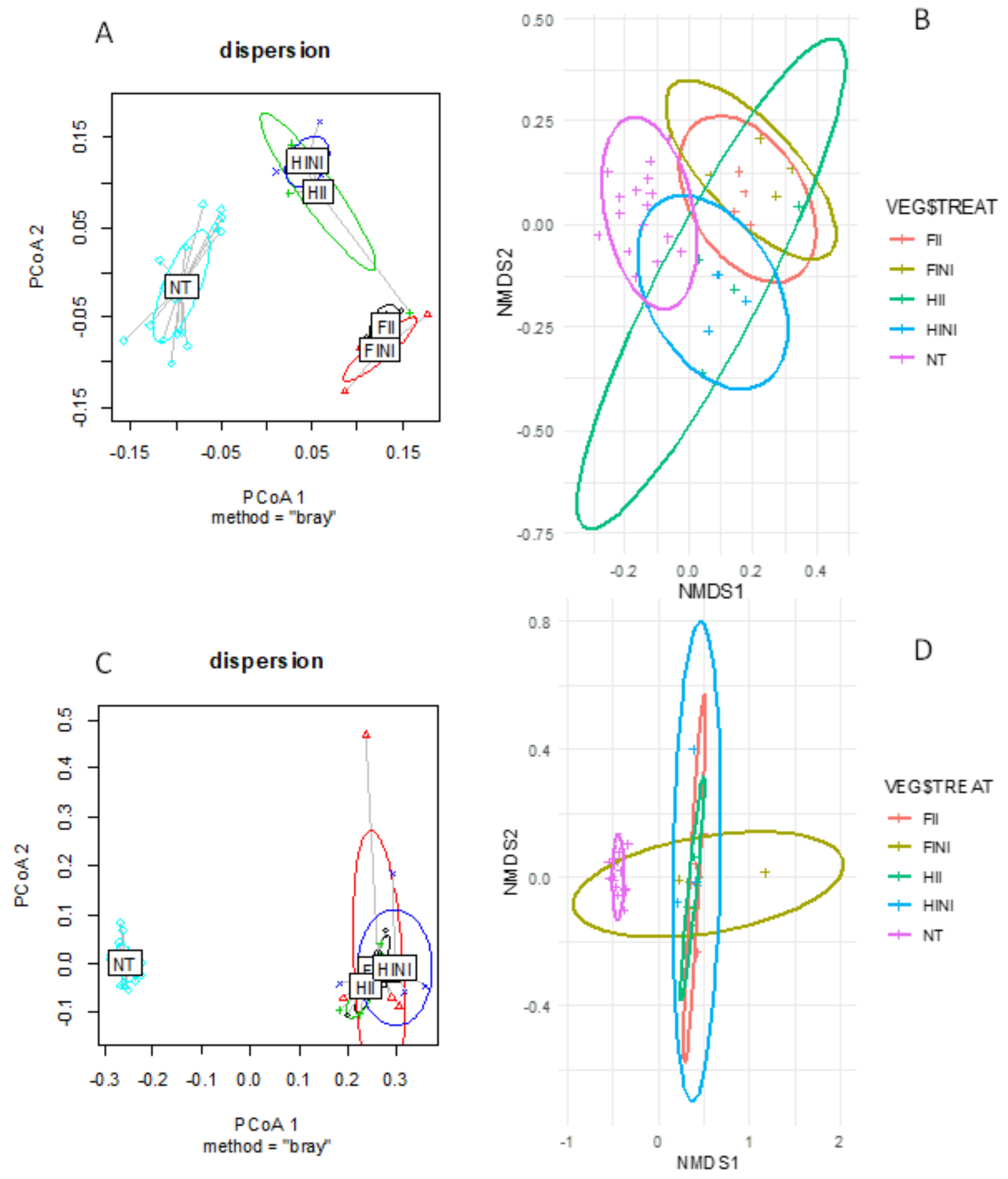

\section{Figure 2}

Principal Coordinate Analysis (PCoA) of the Bray-Curtis distance matrix for bacteria (A) and fungi (C) communities and Nonmetric Multidimensional Scaling (NMDS) plot of Bray-Curtis dissimilarities for bacteria (B) and fungi (D) communities from vineyard soils subjected to different irrigation amounts (FI, Full Irrigated or $\mathrm{HI}$, Half Irrigated), AMF inoculation (I, inoculated; or NI, non-inoculated) and their combinations. 


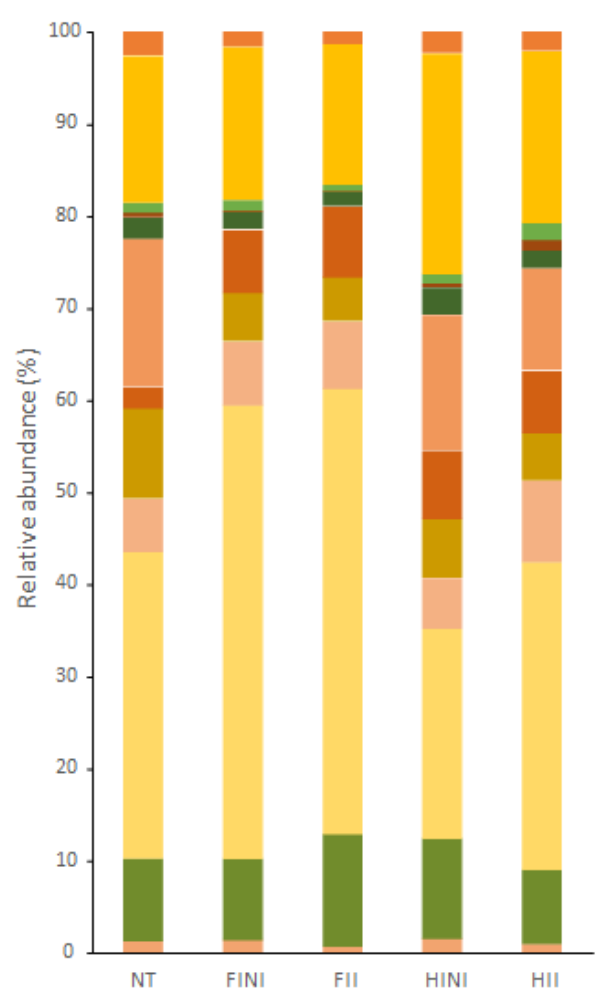

A
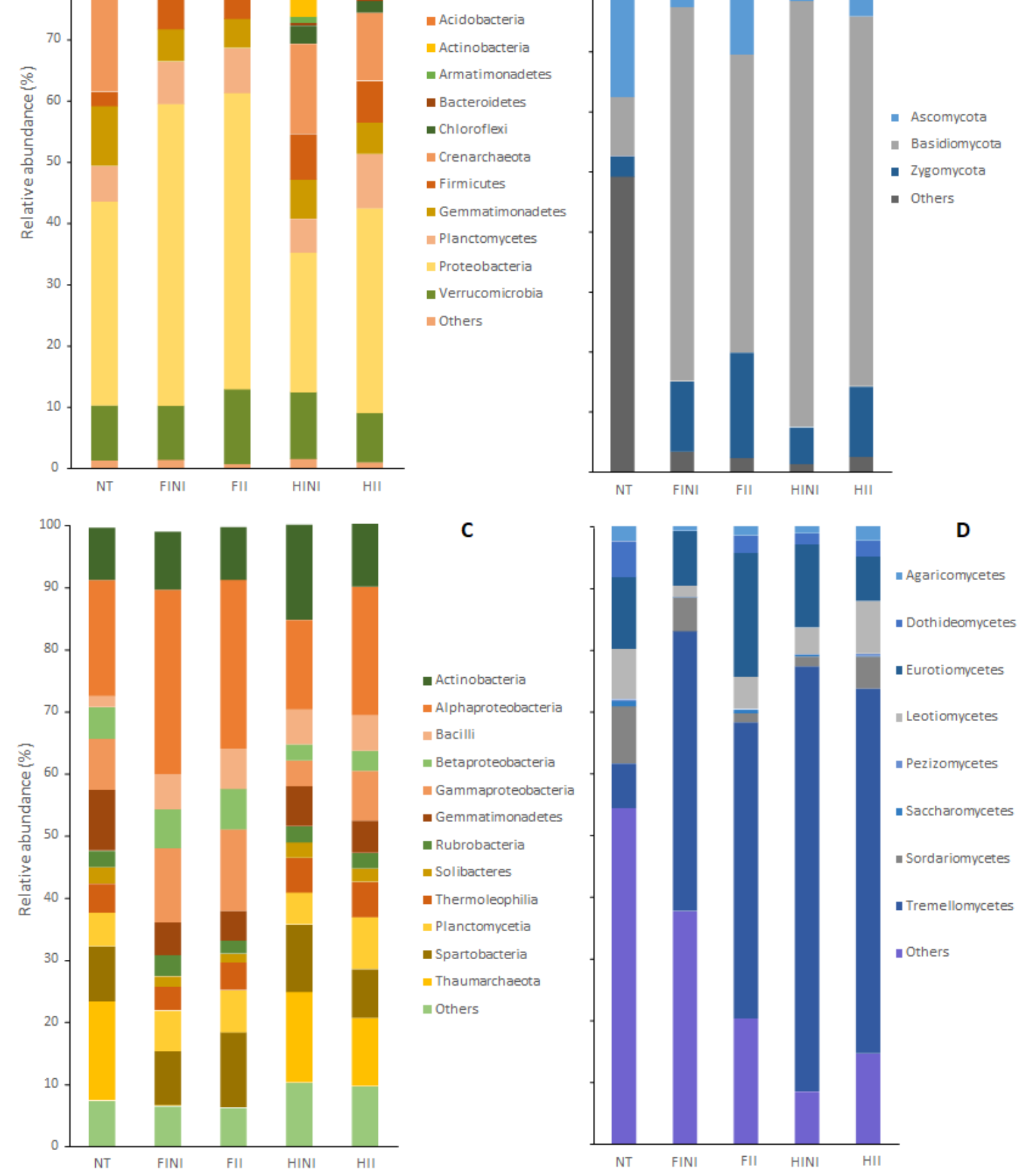

Figure 3

Bacterial and fungal community composition found in plots before (NT) and after treatments, different irrigation amounts (FI, Full Irrigated or HI, Half Irrigated), AMF inoculation (I, inoculated; or NI, noninoculated), and their combination at the phylum (A, B) and class (C, D) levels. 

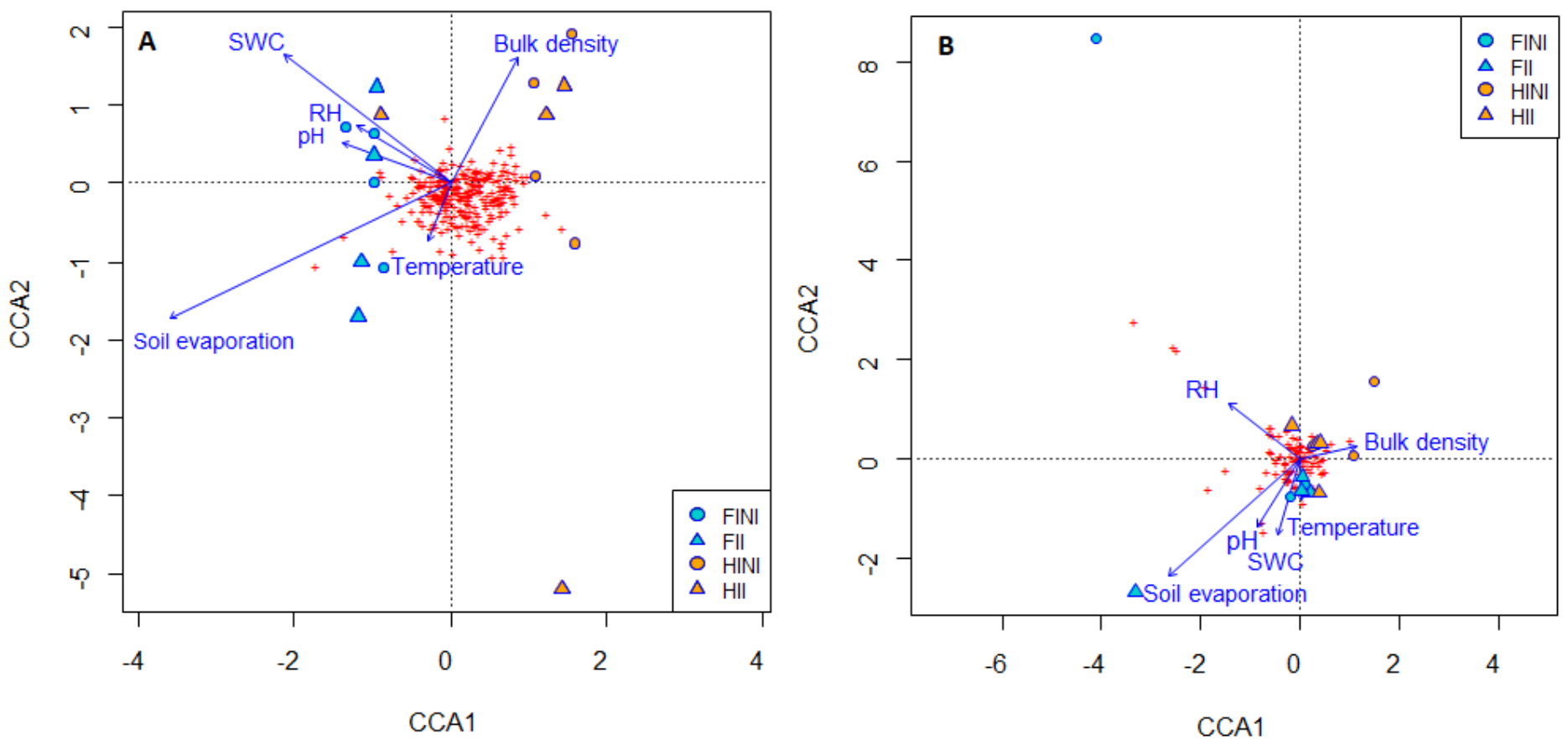

\section{Figure 4}

Canonical Correspondence Analysis (CCA) showing the correlation among soil edaphic factors and bacterial (A) and fungal (B) communities from vineyard soils subjected to different irrigation amounts ( $\mathrm{Fl}$, Full Irrigated or $\mathrm{HI}$, Half Irrigated), AMF inoculation (I, inoculated; or NI, non-inoculated) and their combinations.

A

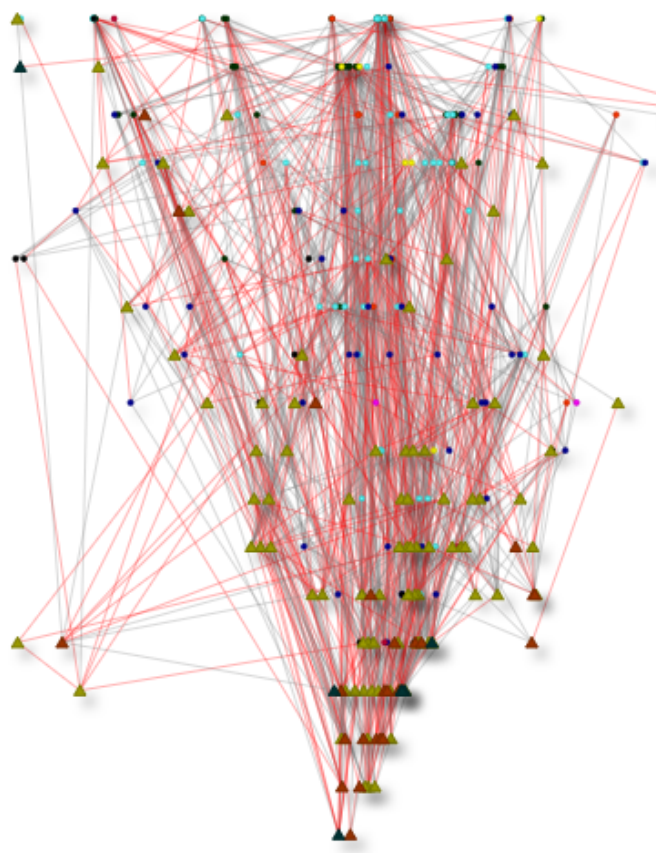

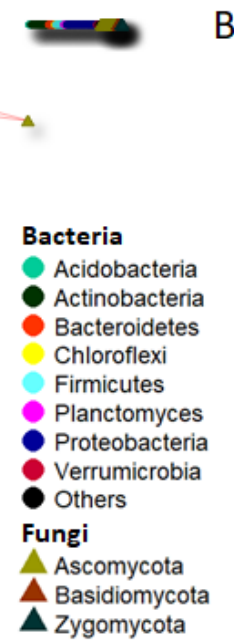

B

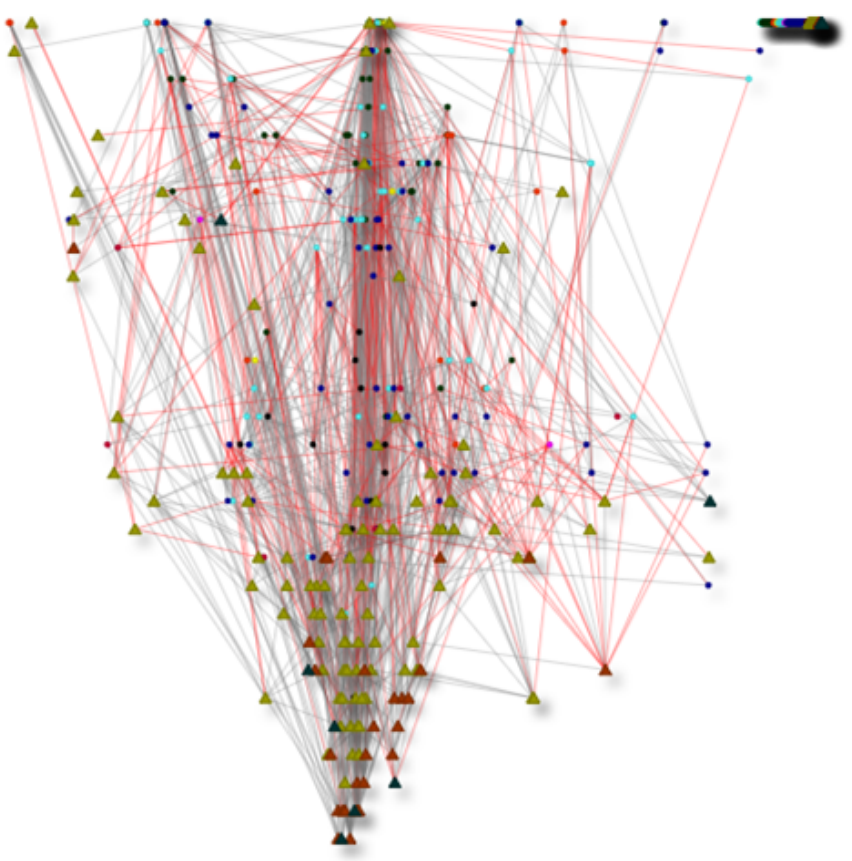




\section{Figure 5}

Significant co-occurrence networks of bulk soil bacterial and fungal communities of Merlot vineyard before treatment application (March 13, A) and after treatment application (June 16, B). The nodes of each network are colored and shaped according to phylum affiliation. The edges connecting the nodes show negative (indicated with red lines) and positive (indicated with grey lines) associations between each species.
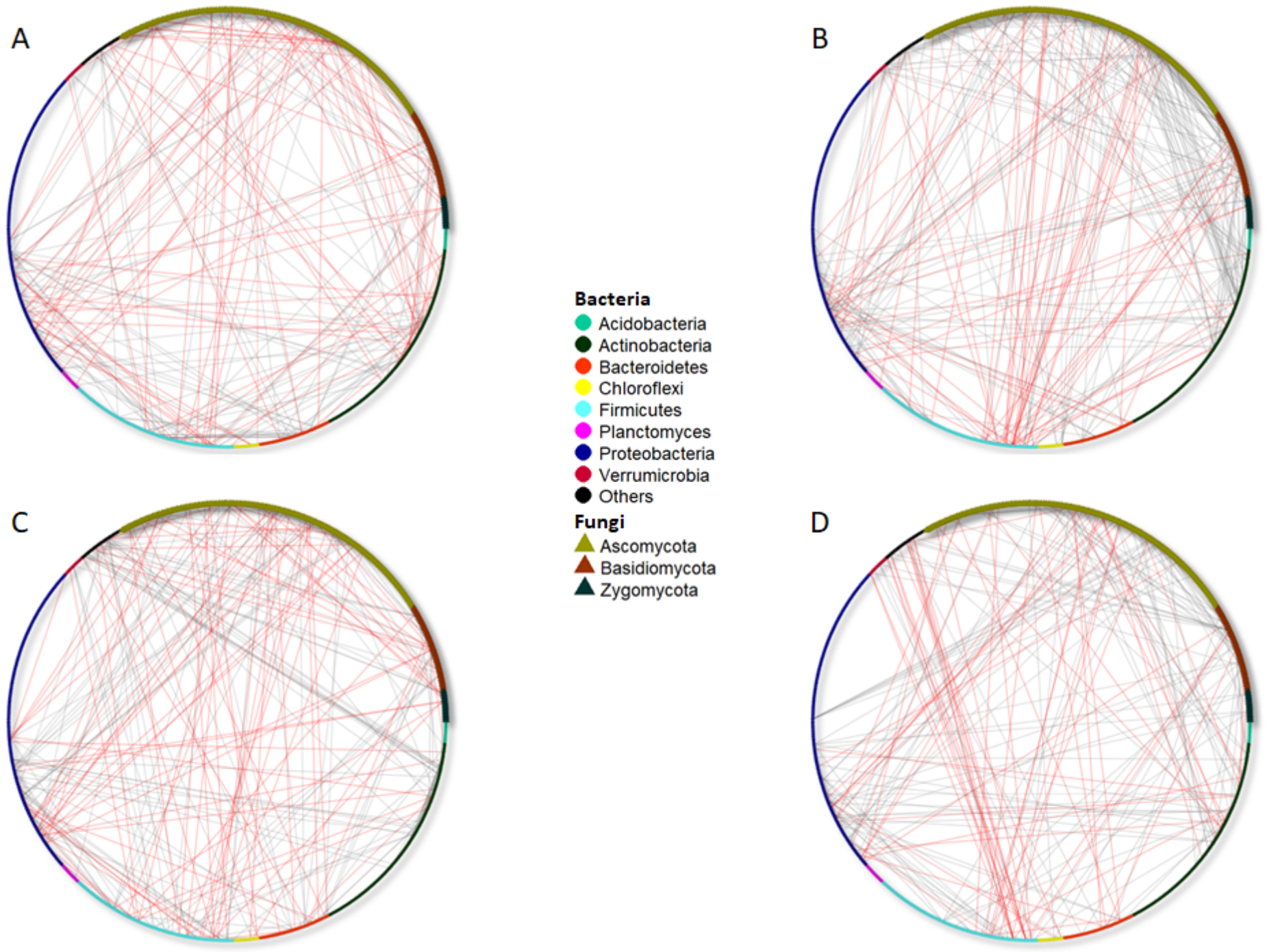

Figure 6

Significant co-occurrence networks of bulk soil bacterial and fungal communities living in the full irrigated $(\mathrm{FI}, \mathrm{A})$, half irrigated $(\mathrm{HI}, \mathrm{B})$, non-inoculated $(\mathrm{NI}, \mathrm{C})$ or inoculated with $\mathrm{AMF}(\mathrm{I}, \mathrm{D})$ plots. The nodes of each network are colored and shaped according to phylum affiliation. The edges connecting the nodes show negative (indicated with red lines) and positive (indicated with grey lines) associations between each species.

\section{Supplementary Files}


This is a list of supplementary files associated with this preprint. Click to download.

- Additionalfile1.pdf

- Additionalfile2.Rcodesandexceldata.zip

- Additionalfile3.xlsx

- Additionalfile4.docx 